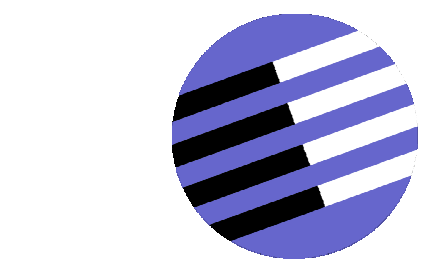

GOVERNANCE AND THE EFFICIENCY

OF ECONOMIC SYSTEMS

GESY

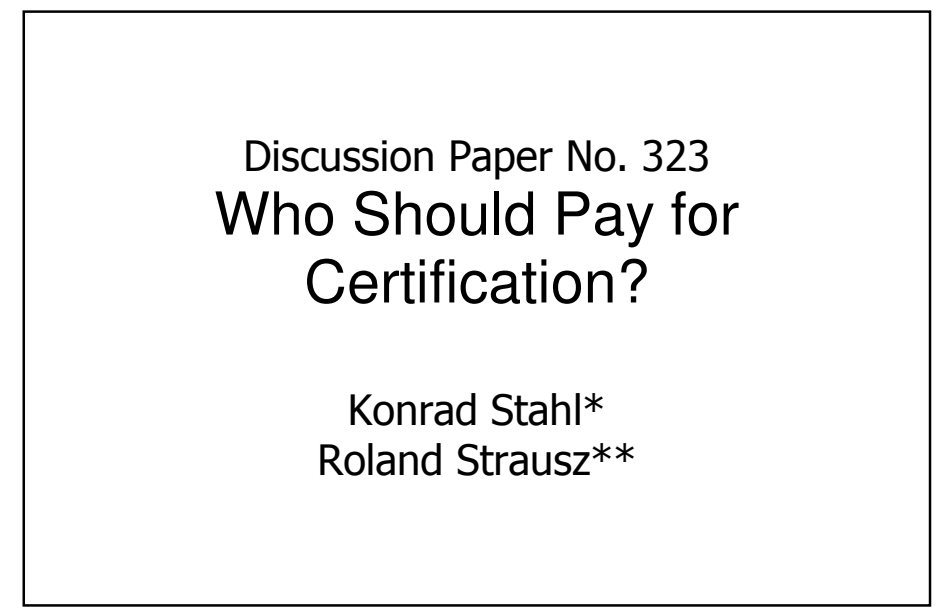

*University of Mannheim, D-68131 Mannheim,

E-mail: kos@econ.uni-mannheim.de

**Humboldt-University at Berlin,

E-mail: strauszr@wiwi.hu-berlin.de

June 2010

Financial support from the Deutsche Forschungsgemeinschaft through SFB/TR 15 is gratefully acknowledged. 


\title{
Who Should Pay for Certification?
}

\author{
Konrad Stahl and Roland Strausz*
}

June 16, 2010

\begin{abstract}
Who does, and who should initiate costly certification by a third party under asymmetric quality information, the buyer or the seller? Our answer - the seller - follows from a nontrivial analysis revealing a clear intuition. Buyer-induced certification acts as an inspection device, seller-induced certification as a signalling device. Seller-induced certification maximizes the certifier's profit and social welfare. This suggests the general principle that certification is, and should be induced by the better informed party. The results are reflected in a case study from the automotive industry, but apply also to other markets - in particular the financial market.
\end{abstract}

JEL Codes: D40, D82, L14, L15

Keywords: asymmetric information, certification, information acquisition, inspection, lemons, middlemen, signaling

\footnotetext{
${ }^{*}$ Contact details: Konrad Stahl, University of Mannheim, D-68131 Mannheim, kos@econ.uni-mannheim.de. Roland Strausz, Humboldt-Universität Berlin, strauszr@wiwi.hu-berlin.de; Research supported by the Deutsche Foschungsgemeinschaft (SFB-TR 15). We are grateful to seminar audiences at the Free University of Berlin, ESSET in Gerzensee, the Hebrew University, the University of Copenhagen, the University of Oxford, Tel Aviv University, the Institute for Advanced Studies Vienna, and in particular Helmut Bester, Eddie Dekel, Leonardo Felli, Hans Gersbach, Zvika Neeman, Andrea Prat, Frank Rosar, and Lucy White for very constructive comments.
} 


\section{Introduction}

In many, if not most markets, the seller of a good knows better its quality than the buyer. Often that seller cannot credibly convey it to the buyer. In this case, an independent expert may be called for to certify the product's quality. In principle, there is demand for certification from both sides of the market. The seller may ask for certification in order to sell at an appropriately high price if the commodity is of high quality. The buyer may ask for certification because she does not want to buy a low quality commodity at a high price.

So does it make a difference whether the buyer or the seller initiates certification? The spontaneous answer seems no. We show, however, that it does. The reason is that the economic role of certification differs drastically, depending on whether the informed or the uninformed party initiates it.

Our question has both a positive and a normative component: Is it more profitable for the certifier to sell its service to the seller, or the buyer? Is its choice appropriate from a welfare point of view?

The answers to these questions are not obvious. It is unclear a priori to whom certification is more valuable and therefore, from whom the certifier can extract more rents. Moreover, it is unclear whether its rent extraction leads to a socially desirable outcome.

Our answers are, nevertheless, unequivocal. Seller-induced certification is more profitable to the certifier - and it is preferred from a welfare point of view. Arriving at these answers is not trivial, because the economic role of certification depends crucially on who initiates it. If the buyer wants to check the seller's claim about quality implicit in his price quotation, certification plays the role of an inspection device. By contrast, if the seller wants to prove high product quality to the buyer, certification induced by him plays the role of a signalling device.

We identify and compare these two different roles of certification, and show how they lead to two fundamentally different economic games. When the buyer initiates certification, buyer and seller play an inspection game with a typical mixed strategy equilibrium. The certifier then picks a price for certification that maximizes its revenue in the mixed strategy equilibrium of the subsequent inspection game. By contrast, when the seller initiates certification, the buyer and the seller play a signalling game. The certifier then 
also picks a certification price that maximizes its revenue, but the price must ensure that certification is an effective signalling device in that it separates high quality from low quality sellers. Hence, the certifier must not only ensure that the price of certification is low enough so that the high quality producer wants to signal high quality via certification, but also high enough so that the low quality seller does not find it worthwhile to buy certification and mimic the high quality seller.

In our baseline analysis, extended and shown to be robust later, we show that the equilibrium outcome in the signalling game is the more efficient one relative to the equilibrium outcome in the inspection game. The reason is that the mixed strategy equilibria of the inspection game yield two inefficiencies; first, certification is sometimes also demanded for the low quality good; and second, the low quality good is not always traded. Hence, in the inspection game, certification is sometimes wasteful, and gains from trade are not always exhausted. By contrast, in the signaling game, certification is demanded only if the good is of high quality, and the good is sold no matter its quality, so gains from trade are fully exploited. We therefore conclude that seller-induced certification is not only socially more desirable, but also preferable to the certifier.

As to the literature on certification, Viscusi (1978) was the first to point out formally that, in Akerlof's (1970) lemons market, there exist gains of trade for an external certifier, who reduces asymmetric information by providing quality certification. ${ }^{1}$ Biglaiser (1993) extends this result to a dynamic adverse selection setting, and derives conditions under which an expert improves upon welfare by taking possession of the good(s) and trading with the typical buyer. Because the expert acts more as a middleman than as a certifier, the model differs from ours. Faulhaber and Yao (1989) focus on how, in a dynamic framework, the possibility of certification impacts concerns of reputation. We do not address reputation. Albano and Lizzeri (2001) consider a moral hazard problem and show how certification can provide the correct incentives for the production of high but costly quality. Yet unlike in our

\footnotetext{
${ }^{1}$ See also De and Nabar (1991), who point out that inaccurate certification technologies may yield quantitatively different results than the perfect certification framework as in Viscusi (1978).
} 
model, the certifier sells, by assumption, only to the producer.

More in line with our research questions, Fasten and Hofmann (2010) discuss the provision of certification to a buyer or a seller, but concentrate on asymmetries in information disclosure: The seller wants public information, the buyer private one. These issues do not arise in our context. Bouvard and Levy (2009) show that in spite of reputation effects involved in certification, the certifier does not necessarily fully disclose information, an aspect not discussed in our comparison.

We follow the aforementioned literature in the assumption that certifiers reveal honestly all their information. Yet there is also a literature on the strategic disclosure of a certifier's information and straight-out fraudulent experts. Lizzeri (1999) focuses on the strategic manipulation of information by a monopolistic certifier and shows that, in its quest of maximizing returns, the certifier minimizes the amount of information provided. Guerra (2001) demonstrates in a slightly modified version that more than a minimal information serves that objective. Peyrache and Quesada (2004) extend Lizzeri's analysis of the strategic disclosure of information by certifiers, to include reputation and differentiation effects between sellers.

Wolinsky (1993) shows how buyers' search for multiple opinions disciplines fraudulent certifiers. Emons (1997) discusses whether in markets for experts, the market mechanism induces non-fraudulent behavior. Strausz (2004) discusses how reputation in a repeated game can induce non-fraudulent behavior even if a seller can bribe a certifier to certify dishonestly. He also shows that honest certification exhibits economies of scale and constitutes a natural monopoly. ${ }^{2}$ While we can use the latter result in our model to justify our assumption of a certifier monopoly, this strand of literature is very different in spirit and intention to ours.

The rest of the paper is organized as follows. In the next Section 2, we describe the model. In Section 3, we derive the results for buyer-induced certification. Section 4 contains the results for seller-induced certification as well as the comparison between the two from the point of view of the

\footnotetext{
${ }^{2}$ See Hvide (2004) for a model with several certifiers, who compete in prices but are ranked by the difficulty at which their test is passed. Broadly speaking the author shows that the matching of sellers and certifiers is assortative.
} 
certifier. In Section 5 we evaluate that outcome from a welfare point of view. Section 6 we discuss many extensions of our baseline model and show the results to be robust. In Section 7 we discuss empirical examples involving certification. Whilst the primary one is taken from upstream supplier-buyer relationships in the automotive industry, there are many other applications - in particular to the financial industry, with our results leading to a clear policy recommendation. We summarize and conclude with Section 8. All proofs are relegated to the Appendix.

\section{Model}

Consider a seller offering one unit of a good at price $p$ whose quality, before certification, is revealed only to him and is unobservable to a buyer. From the buyer's point of view, the seller's quality is high, $q_{h}$, with probability $\lambda$ and low, $q_{l}>0$, with probability $1-\lambda$, where $\Delta q \equiv q_{h}-q_{l}>0 .{ }^{3}$ The good's quality is identified with the buyer's willingness to pay. The risk neutral buyer is therefore willing to pay up to a price that equals expected quality $\bar{q} \equiv \lambda q_{h}+(1-\lambda) q_{l}$. If not buying at all, his reservation payoff is zero. The high quality seller has a production cost $c_{h}>0$, and the low quality seller has a production cost $c_{l}=0$. If not producing and selling the seller's reservation payoff is also zero.

We assume that the high quality good delivers higher economic rents: $q_{h}-c_{h}>q_{l}-c_{l}=q_{l}>0$. Moreover, the cost of producing a high quality product exceeds the average quality, $c_{h}>\bar{q}$. This creates a lemon's problem and leads to adverse selection: without certification, a high quality seller would not offer his good to the market, and thus the market outcome with informational asymmetry would be inefficient. Without the informational asymmetry, however, the high quality seller could sell his good for the price $q_{h}>c_{h}$. Consequently, the high quality seller has demand for certification that reveals the good's true quality to the buyer. Clearly, the high quality seller is willing to pay the certifier at most $q_{h}-c_{h}$.

Yet the buyer has also demand for certification. Whenever the seller

\footnotetext{
${ }^{3}$ This implies that $\lambda$ is bounded away from 1 .
} 
quotes a price higher than that appropriate for the low quality good, the buyer may demand certification ascertaining that the good has indeed high quality, so that a higher price is justified.

Summarizing, both the buyer and the seller have a demand for certification. For a monopolistic certifier this brings the question as to whom he should offer his services. ${ }^{4}$ The certifier has the technology to perfectly detect the seller's quality at a cost $c_{c} \in\left[0, q_{h}-c_{h}\right)$ and to announce it publicly.

The certifier's problem is as follows. In an initial stage (not modeled here to keep matters as simple as possible), he has to decide whether to offer his services to the buyer or the seller. After this decision, he sets a price $p_{c}$ at which the buyer or the seller, respectively, can obtain certification. If not offering certification at all, his reservation payoff is zero. We focus on honest certification where the certifier cannot be bribed.

Our research question is twofold, namely whether the monopolistic certifier is better off servicing the uninformed buyer or the informed seller, and whether his decision conforms to the sum of consumer and producer surplus, a standard welfare criterion. In order to answer these questions, we separately study "buyer-induced", and "seller-induced" certification, and contrast their outcomes from both the certifier's and a social welfare point of view.

\section{Buyer-Induced Certification}

Here we consider the certification problem when induced by the buyer. Before analyzing the formal model, it is helpful to provide an intuition on the role of certification and the certifier's motive in this setup.

Buyer-induced certification enables the buyer to check the seller's quality claim. In particular, certification offers the buyer protection against a

\footnotetext{
${ }^{4}$ For obvious reasons, the certifier cannot sell to both parties at the same time. In Section 7 , we consider empirical cases exactly reflecting this structure.
} 
low quality seller who pretends to have high quality. From the buyer's perspective, therefore, certification is an inspection device to detect low quality sellers.

The game underlying buyer-induced certification, therefore, is an inspection game. A mixed strategy equilibrium is typical for this type of game. Indeed, a pure strategy equilibrium in which the buyer never buys certification cannot exist, because it would give the low quality seller an incentive to claim high quality - yet against this claim the buyer would have a strong incentive to buy certification. Likewise, an equilibrium in which the buyer always buys certification cannot exist either, because it keeps the low quality seller from claiming high quality - yet against such behavior certification is only wasteful for the buyer. Consequently, we typically have a mixed strategy equilibrium, where the low quality seller cheats with some probability and claims to offer high quality, and the buyer certifies with some probability when the seller claims to have high quality.

Hence, buyer-induced certification plays the role of reducing cheating. The buyer's demand for certification will therefore be high when the problem of cheating is large. This reasoning suggests that a monopolistic certifier, who targets his services towards the buyer, will choose a certification price that maximizes the buyer's cheating problem.

A closer look reveals that the buyer's cheating problem depends on two factors: the buyer's uncertainty and the seller's price quotation. First, the buyer's cheating problem is the bigger the less certain she is about the true quality offered by the seller. Second, checking true quality through certification is especially worthwhile for intermediate prices of the good. Indeed, for a low price the buyer would not lose much from simply buying the good uncertified. By contrast, when the price is high, the buyer would not lose much from not buying the good at all. Hence, the buyer's willingness to pay for certification is largest for intermediate prices that are neither too low nor too high.

To sum up, our intuitive reasoning suggests that under buyer-induced certification a monopolistic certifier will choose his price for certification, $p_{c}$, 
so that it induces high uncertainty for the buyer and an intermediate price for the good. With the ensuing formal analysis we show that this intuition is correct, yet not trivial at all.

With buyer-induced certification, the parties play the following game:

$\mathrm{t}=1$ The certifier sets a price $p_{c}$ for his service.

$\mathrm{t}=2$ Nature selects the quality $q_{i}, i \in\{l, h\}$, of the good offered, and conveys it to the seller.

$\mathrm{t}=3$ The seller offering the good of quality $q_{i}$ at $\operatorname{cost} c_{i}$ decides about the price $p$ at which he offers the good.

$\mathrm{t}=4$ The buyer decides whether or not to demand certification of the good.

$\mathrm{t}=5$ The buyer decides whether or not to buy the good.

Note that we assume that if the seller $q_{i}$ sets a price in stage 3 , he incurs the production $\operatorname{cost} c_{i}$ for sure, even though the buyer may decide not to buy the good in stage 5 . This assumption is natural under several forms of certification.

First, certification may mean that the certifier inspects the actual good the buyer is interested in. In this case, the good must already be produced in order for the certifier to inspect it, and the seller must therefore have incurred the production cost even if the buyer decides not to acquire it. A second possibility is that the certifier determines the seller's product quality by inspecting his production facility, and certifying his production technology. In this case, the production cost $c_{h}$ may be interpreted as a fixed cost that differs between high and low quality sellers. Under both interpretations, the seller incurs the cost even if the buyer, in the end, does not buy the product.

We focus on the Perfect Bayesian Equilibria (PBE) of the game described above. Note that after the certifier has set its price $p_{c}$, a proper subgame, $\Gamma\left(p_{c}\right)$, starts with nature's decision about the quality of the seller's product. 
The subgame $\Gamma\left(p_{c}\right)$ is a signalling game where the seller's price $p$ may or may not reveal his private information about the quality of the good.

In the subsequent analysis, we first consider the PBE of the subgame $\Gamma\left(p_{c}\right)$. A PBE specifies three components: First, the seller's pricing strategy as a function of the good's type $q_{i}$; second, the buyer's belief $\mu(p)$ after observing the price $p$; third, the buyer's behavior; in particular whether or not to buy certification and the actual good.

We allow the seller to randomize over prices. In order to circumvent measure-theoretical complications, we assume that the seller can randomize over an infinite but countable set. Consequently, we can express the strategy of the seller of quality $q_{i}$ by the function $\sigma_{i}: R_{+} \rightarrow[0,1]$ with the interpretation that $\sigma_{i}\left(p_{j}\right)$ denotes the probability that the seller with quality $q_{i}$ chooses the price $p_{j}$. Thus, for both $i \in\{h, l\}$,

$$
\sum_{j} \sigma_{i}\left(p_{j}\right)=1
$$

The buyer's decisions are based on his belief specified as a function $\mu: \mathbb{R}_{+} \rightarrow$ $[0,1]$ with the interpretation that, after observing price $p$, the buyer believes that the seller is of type $q_{h}$ with probability $\mu(p)$.

We can express the buyer's behavior after observing the price $p$ and possessing some belief $\mu$ by the following six actions:

1. Action $s_{n n}$ : The buyer does not buy certification nor buy the good. This action yields the payoff

$$
U\left(s_{n n} \mid p, \mu\right)=0 .
$$

2. Action $s_{n b}$ : The buyer does not buy certification, but buys the product. This action yields the expected payoff

$$
U\left(s_{n b} \mid p, \mu\right)=\mu q_{h}+(1-\mu) q_{l}-p .
$$

3. Action $s_{c h}$ : The buyer buys certification and buys the product only when the certifier reveals high quality. This action yields the expected payoff

$$
U\left(s_{c h} \mid p, \mu\right)=\mu\left(q_{h}-p\right)-p_{c} .
$$


4. Action $s_{c b}$ : The buyer buys certification and buys the product irrespective of the outcome of certification. This action yields the expected payoff

$$
U\left(s_{c b} \mid p, \mu\right)=\mu\left(q_{h}-p\right)+(1-\mu)\left(q_{l}-p\right)-p_{c} .
$$

Clearly, $U\left(s_{c b} \mid p, \mu\right)<U\left(s_{n b} \mid p, \mu\right)$ for any $p_{c}>0$ so that the action $s_{n b}$ dominates the action $s_{c b}$.

5. Action $s_{c l}$ : The buyer buys certification and buys the product only when the certifier reveals low quality. This action yields the expected payoff

$$
U\left(s_{c l} \mid p, \mu\right)=(1-\mu)\left(q_{l}-p\right)-p_{c} .
$$

Clearly, $U\left(s_{c l} \mid p, \mu\right) \leq U\left(s_{n b} \mid p, \mu\right)$ for $p \leq q_{h}$ and $U\left(s_{c l} \mid p, \mu\right) \leq U\left(s_{n n} \mid p, \mu\right)$ for $p>q_{h}$. Hence, also the action $s_{c l}$ is weakly dominated.

6. Action $s_{c n}$ : The buyer demands certification and does not buy the product. This action yields the expected payoff

$$
U\left(s_{c n} \mid p, \mu\right)=-p_{c}
$$

Clearly, $U\left(s_{c n} \mid p, \mu\right)<U\left(s_{n n} \mid p, \mu\right)$ for any $p_{c}>0$ so that the action $s_{c n}$ is dominated.

To summarize, only the first three actions $s_{n n}, s_{n b}, s_{c h}$ are not (weakly) dominated for some combination $(p, \mu)$. The intuition is straightforward: the role of certification is to enable the buyer to discriminate between high and low quality. It is therefore only worthwhile to buy certification when the buyer uses it to screen out bad quality. ${ }^{5}$

In the following, we delete the weakly dominated actions from the buyer's action space. Consequently, we take the buyer's action space as $S \equiv\left\{s_{n n}, s_{n b}, s_{c h}\right\}$. Since we want to allow the buyer to use a mixed strategy, we let $\sigma(s \mid p, \mu) \in$

\footnotetext{
${ }^{5}$ Observe that the strategy $s_{c h}$ is not renegotiation proof, because even after certification has revealed low quality, gains could be realized by trading the low quality product. In Section 6, we will consider the simple extension to include renegotiation.
} 
$[0,1]$ represent the probability that the buyer takes action $s \in S=\left\{s_{n n}, s_{n b}, s_{c h}\right\}$ given price $p$ and belief $\mu$. Thus

$$
\sum_{s \in S} \sigma(s \mid p, \mu)=1
$$

A PBE in our subgame $\Gamma\left(p_{c}\right)$ can now be described more specifically: it is a tuple of functions $\left\{\sigma_{l}, \sigma_{h}, \mu, \sigma\right\}$ satisfying the following three equilibrium conditions. First, seller type $i$ 's pricing strategy $\sigma_{i}$ must be optimal with respect to the buyer's strategy $\sigma$. Second, the buyer's belief $\mu$ must be consistent with the sellers' pricing strategy, whenever possible. Third, the buyer's strategy $\sigma$ must be a best response given the observed price $p$ and her beliefs $\mu$.

We start our analysis of the Perfect Bayesian Equilibria of $\Gamma\left(p_{c}\right)$ by studying the third requirement: the optimality of the buyer's strategy given a price $p$ and beliefs $\mu$.

Fix a price $\bar{p}$ and a belief $\bar{\mu}$. Then the pure strategy $s_{n n}$ is a best response whenever $U\left(s_{n n} \mid \bar{p}, \bar{\mu}\right) \geq U\left(s_{n b} \mid \bar{p}, \bar{\mu}\right)$ and $U\left(s_{n n} \mid \bar{p}, \bar{\mu}\right) \geq U\left(s_{c h} \mid \bar{p}, \bar{\mu}\right)$. It follows that the strategy $s_{n n}$ is a best response whenever

$$
(\bar{p}, \bar{\mu}) \in S\left(s_{n n} \mid p_{c}\right) \equiv\left\{(p, \mu) \mid p \geq \mu q_{h}+(1-\mu) q_{l} \wedge p_{c} \geq \mu\left(q_{h}-p\right)\right\} .
$$

Likewise, the pure strategy $s_{n b}$ is (weakly) preferred whenever $U\left(s_{n b} \mid \bar{p}, \bar{\mu}, p_{c}\right) \geq$ $U\left(s_{n n} \mid \bar{p}, \bar{\mu}, p_{c}\right)$ and $U\left(s_{n b} \mid \bar{p}, \bar{\mu}, p_{c}\right) \geq U\left(s_{c h} \mid \bar{p}, \bar{\mu}, p_{c}\right)$. It follows that the strategy $s_{n b}$ is a best response whenever

$$
(\bar{p}, \bar{\mu}) \in S\left(s_{n b} \mid p_{c}\right) \equiv\left\{(p, \mu) \mid p \leq \mu q_{h}+(1-\mu) q_{l} \wedge p_{c} \geq(1-\mu)\left(p-q_{l}\right)\right\} .
$$

Finally, the pure strategy $s_{c h}$ is (weakly) preferred whenever $U\left(s_{c h} \mid \bar{p}, \bar{\mu}, p_{c}\right) \geq$ $U\left(s_{n n} \mid \bar{p}, \bar{\mu}, p_{c}\right)$ and $U\left(s_{c h} \mid \bar{p}, \bar{\mu}, p_{c}\right) \geq U\left(s_{n b} \mid \bar{p}, \bar{\mu}, p_{c}\right)$. It follows that the strategy $s_{c h}$ is a best response whenever

$$
(\bar{p}, \bar{\mu}) \in S\left(s_{c h} \mid p_{c}\right) \equiv\left\{(p, \mu) \mid p_{c} \leq \mu\left(q_{h}-p\right) \wedge p_{c} \leq(1-\mu)\left(p-q_{l}\right)\right\} .
$$

Since a mixed strategy is only optimal if it randomizes among those pure strategies that are a best response, we arrive at the following result: 


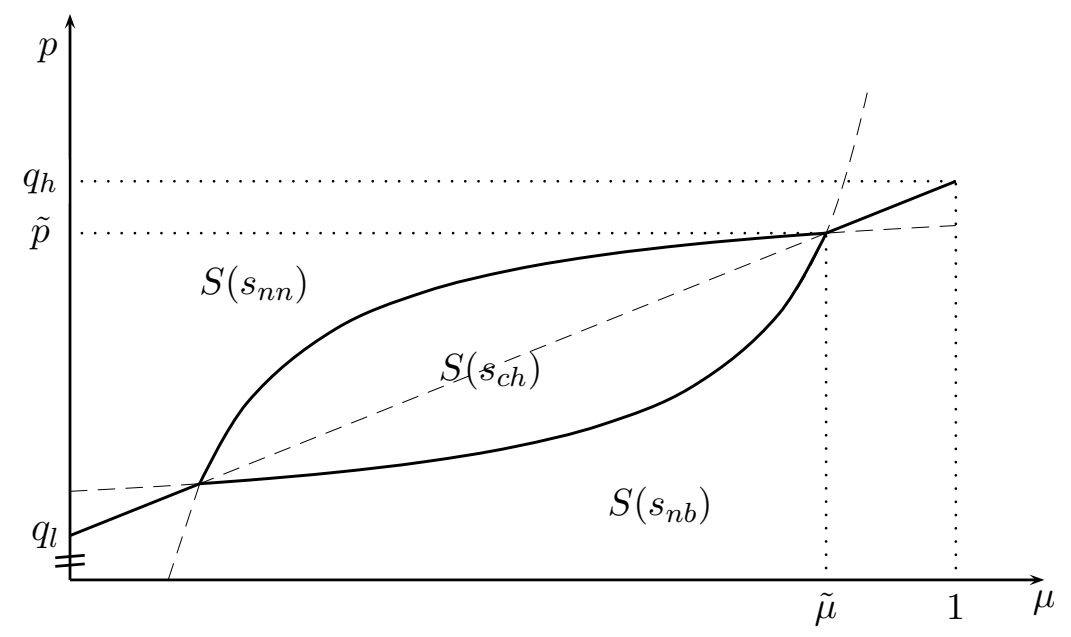

Figure 1: Buyer's buying behavior for given $p_{c}<\Delta q / 4$.

Lemma 1 In any Perfect Bayesian Equilibrium $\left(\sigma_{l}^{*}, \sigma_{h}^{*}, \mu^{*}, \sigma^{*}\right)$ of the subgame $\Gamma\left(p_{c}\right)$ we have for any $s \in S=\left\{s_{n n}, s_{n b}, s_{c h}\right\}$,

$$
\sigma^{*}(s \mid p, \mu)>0 \Rightarrow\left(p, \mu^{*}(p)\right) \in S\left(s \mid p_{c}\right)
$$

Figure 1 illustrates the buyer's behavior for a given certification price $p_{c}$. For low prices $p$ the buyer buys the good uncertified, $(p, \mu) \in S\left(s_{n b}\right)$, whereas for high prices $p$ the buyer refrains from buying, $(p, \mu) \in S\left(s_{n n}\right)$. As long as $p_{c}<\Delta q / 4$ there is an intermediate range of prices $p$ and beliefs $\mu$ such that the buyer demands certification, i.e. $(p, \mu) \in S\left(s_{c h}\right)$. In this case, the buyer only buys the product when certification reveals that it has high quality. Intuitively, the buyer demands certification to ensure that the highly priced product is indeed of high quality. Note that apart from points on the thick, dividing lines, the buyer's optimal buying behavior of both certification services and the product is uniquely determined, and mixing does not take place.

For future reference we define

$$
\tilde{p} \equiv\left(q_{h}+q_{l}+\sqrt{\Delta q\left(\Delta q-4 p_{c}\right)}\right) / 2
$$


and

$$
\tilde{\mu} \equiv\left(1+\sqrt{1-4 p_{c} / \Delta q}\right) / 2 .
$$

Note that if the seller prices at $\tilde{p}$ and the buyer has beliefs $\tilde{\mu}$, the buyer is indifferent between all three decisions namely not to buy the good, $s_{n n}$, to buy the good uncertified, $s_{n b}$, or to buy the good only after it has been certified as high quality, $s_{c h}$.

We previously argued that the monopolistic certifier benefits from high buyer uncertainty and an intermediate price of the good. We now can give precision to this statement. The buyer's willingness to pay for certification is the difference between her payoff from certification and the next best alternative, namely either to buy the good uncertified, or to not buy the good at all. More precisely, given her beliefs are $\mu$, the difference in the buyer's expected payoffs between buying the high quality good when certified and buying any good uncertified is

$$
\Delta U^{1} \equiv \mu\left(q_{h}-p\right)-(\bar{q}-p)
$$

Similarly, the difference in the buyer's expected payoffs between buying the good only when certified and buying the good not at all is

$$
\Delta U^{2}=\mu\left(q_{h}-p\right)
$$

Hence, the buyer's willingness to pay for certification is maximized for a price $\hat{p}$ and a belief $\hat{\mu}$ that solves

$$
\max _{p, \mu} \min \left\{\Delta U^{1}, \Delta U^{2}\right\}
$$

The solution is $\hat{\mu}=1 / 2$ and $\hat{p}=\left(q_{h}+q_{l}\right) / 2$. We later demonstrate that, with buyer-induced certification, the certifier chooses a price $p_{c}$ for certification to induce this outcome as closely as possible.

Next, we address the optimality of type $i$ seller's strategy $\sigma_{i}(p)$. For a given strategy $\sigma$ of the buyer and a fixed belief $\mu$, a seller with quality $q_{h}$ expects the following payoff from setting a price $p$ :

$$
\Pi_{h}(p, \mu \mid \sigma)=\left[\sigma\left(s_{n b} \mid p, \mu\right)+\sigma\left(s_{c h} \mid p, \mu\right)\right] p-c_{h}
$$


A specific strategy $\sigma_{h}$ yields seller $q_{h}$, therefore, an expected profit of

$$
\bar{\Pi}_{h}\left(\sigma_{h}\right)=\sum_{i} \sigma_{h}\left(p_{i}\right) \Pi_{h}\left(p_{i}, \mu\left(p_{i}\right) \mid \sigma\right) .
$$

Likewise, a seller with quality $q_{l}$ obtains the payoff

$$
\Pi_{l}(p, \mu \mid \sigma)=\sigma\left(s_{n b} \mid p, \mu\right) p
$$

and any strategy $\sigma_{l}$ yields

$$
\bar{\Pi}_{l}\left(\sigma_{l}\right)=\sum_{i} \sigma_{l}\left(p_{i}\right) \Pi_{l}\left(p_{i}, \mu\left(p_{i}\right) \mid \sigma\right) .
$$

It follows that in a $\operatorname{PBE}\left(\sigma_{h}^{*}, \sigma_{l}^{*}, \mu^{*}, \sigma^{*}\right)$ the high quality seller $q_{h}$ and the low quality seller $q_{l}$ 's payoffs, respectively, are

$$
\Pi_{h}^{*}=\sum_{i} \sigma_{h}^{*}\left(p_{i}\right) \Pi_{h}\left(p_{i}, \mu^{*}\left(p_{i}\right) \mid \sigma^{*}\right) \text { and } \Pi_{l}^{*}=\sum_{i} \sigma_{l}^{*}\left(p_{i}\right) \Pi_{l}\left(p_{i}, \mu^{*}\left(p_{i}\right) \mid \sigma^{*}\right),
$$

respectively.

The next lemma makes precise the intuitive result that the seller's expected profits increase when the buyer has more positive beliefs about the good's quality.

Lemma 2 In any $\operatorname{PBE}\left(\sigma_{l}^{*}, \sigma_{h}^{*}, \mu^{*}, \sigma^{*}\right)$ of the subgame $\Gamma\left(p_{c}\right)$ with $p_{c}>0$ the payoffs $\Pi_{h}\left(p, \mu \mid \sigma^{*}\right)$ and $\Pi_{l}\left(p, \mu \mid \sigma^{*}\right)$ are non-decreasing in $\mu$.

Seller type $i$ 's pricing strategy $\sigma_{i}$ is an optimal response to the buyer's behavior $\left(\sigma^{*}, \mu^{*}\right)$ exactly if, for any $p^{\prime}$, we have

$$
\sigma_{i}^{*}(p)>0 \Rightarrow \Pi_{i}\left(p, \mu^{*}(p) \mid \sigma^{*}\right) \geq \Pi_{i}\left(p^{\prime}, \mu^{*}\left(p^{\prime}\right) \mid \sigma^{*}\right) .
$$

Because the buyer's beliefs depend on the observed price $p$, it affects the buyer's behavior and, therefore, the belief function $\mu^{*}$ plays a role in condition (2).

Finally, a PBE demands that the buyer's beliefs $\mu^{*}$ have to be consistent with equilibrium play. In particular, they must follow Bayes' rule:

$$
\sigma_{i}^{*}(p)>0 \Rightarrow \mu^{*}(p)=\frac{\lambda \sigma_{h}^{*}(p)}{\lambda \sigma_{h}^{*}(p)+(1-\lambda) \sigma_{l}^{*}(p)} .
$$


The next lemma shows some intuitive implications on PBEs that are due to Bayes' rule. In particular, it shows that the seller, no matter his type, never sets a price below $q_{l}$, and the low quality seller never sets a price above $q_{h}$. The lemma also shows that, in equilibrium, the low quality seller never loses from the presence of asymmetric information, since he can always guarantee himself the payoff $q_{l}$ that he obtains with observable quality. By contrast, the high quality seller loses from the presence of asymmetric information; his payoff is strictly smaller than $q_{h}-c_{h}$.

Lemma 3 In any $\operatorname{PBE}\left(\sigma_{l}^{*}, \sigma_{h}^{*}, \mu^{*}, \sigma^{*}\right)$ of the subgame $\Gamma\left(p_{c}\right)$ we have $\left.i\right) \sigma_{l}^{*}(p)=$ 0 for all $p \notin\left[q_{l}, q_{h}\right]$ and $\sigma_{h}^{*}(p)=0$ for all $p<q_{l}$; ii) $\Pi_{l}^{*} \geq q_{l}$; iii) $\Pi_{h}^{*}<q_{h}-c_{h}$.

As is well known, the concept of Perfect Bayesian Equilibrium places only very weak restrictions on admissible beliefs. In particular, it does not place any restrictions on the buyer's beliefs for prices that are not played in equilibrium; any out-of-equilibrium belief is allowed. However, as is typical for signalling games, without any restrictions on out-of-equilibrium beliefs we cannot pin down behavior in the subgame $\Gamma\left(p_{c}\right)$ to a specific equilibrium. Especially by the use of pessimistic out-of-equilibrium beliefs, one can sustain many pricing strategies in equilibrium.

In order to reduce the arbitrariness of equilibrium play, it is necessary to strengthen the solution concept of PBE by introducing more plausible restrictions on out-of-equilibrium beliefs. Bester and Ritzberger (2001) demonstrate that the following extension of the intuitive criterium of Cho-Kreps suffices to pin down equilibrium play.

Belief restriction (B.R.): A Perfect Bayesian Equilibrium $\left(\sigma_{h}^{*}, \sigma_{l}^{*}, \mu^{*}, \sigma^{*}\right)$ satisfies the Belief Restriction if, for any $\mu \in[0,1]$ and any out-of-equilibrium price $p$, we have

$$
\Pi_{l}(p, \mu)<\Pi_{l}^{*} \wedge \Pi_{h}(p, \mu)>\Pi_{h}^{*} \Rightarrow \mu^{*}(p) \geq \mu .
$$

The belief restriction contains the intuitive criterion of Cho-Kreps as the special case for $\mu=1$. Indeed, the underlying idea of the restriction is to extend the idea behind the Cho-Kreps criterion to a situation where 
a deviation to $p$ is profitable only for the $q_{h}$ seller when the buyer believes that the deviation originates from the $q_{h}$ seller with probability $\mu$. As we may have $\mu<1$, the restriction considers more pessimistic beliefs than the Cho-Kreps criterium. If such a pessimistic belief $\mu$ gives only the $q_{h}$ seller an incentive to deviate, then the restriction requires that the buyer's actual belief should not be even more pessimistic than $\mu$.

The next Lemma establishes characteristics of the equilibrium that are due to the belief refinement (B.R.). It shows that the belief restriction implies that the high quality seller can sell his product for a price of at least $\tilde{p}$.

Lemma 4 Any Perfect Bayesian Equilibrium $\left(\sigma_{l}^{*}, \sigma_{h}^{*}, \mu^{*}, \sigma^{*}\right)$ of the subgame $\Gamma\left(p_{c}\right)$ that satisfies B.R. exhibits i) $\sigma_{h}^{*}(p)=0$ for all $p<\tilde{p}$ and ii) $\Pi_{h}^{*} \geq$ $\tilde{p}-c_{h}$.

By combining the previous two lemmata we are now able to characterize the equilibrium outcome.

Proposition 1 Consider a PBE $\left(\sigma_{l}^{*}, \sigma_{h}^{*}, \mu^{*}, \sigma^{*}\right)$ of the subgame $\Gamma\left(p_{c}\right)$ that satisfies B.R. Then

i) for $\lambda<\tilde{\mu}$ and $c_{h}<\tilde{p}$ it exhibits unique pricing behavior by the seller and unique buying behavior by the buyer. In particular, the high quality seller sets the price $\tilde{p}$ with certainty and the low quality seller randomizes between the price $\tilde{p}$ and $q_{l}$. Observing the price $\tilde{p}$ the buyer buys certification with positive probability. The certifier's equilibrium profit equals

$$
\Pi_{c}\left(p_{c}\right)=\frac{\lambda\left(\tilde{p}-q_{l}\right)}{\tilde{\mu} \tilde{p}}\left(p_{c}-c_{c}\right) .
$$

ii) For $\lambda>\tilde{\mu}$ or $c_{h}>\tilde{p}$ we have $\Pi_{c}\left(p_{c}\right)=0$ in any equilibrium.

iii) For $\lambda \leq \tilde{\mu}$ and $c_{h} \leq \tilde{p}$ there exists an equilibrium outcome, in which the certifier's profits equal expression (4).

The Proposition shows that the buyer and the low quality seller play the mixed strategies that reflect the typical outcome of an inspection game. Indeed, by choosing the low price $q_{l}$ a low quality seller honestly signals his low quality. In contrast, we may interpret a low quality seller, who sets a 
high price $\tilde{p}$, as trying to cheat. Hence, whenever the buyer observes the price $\tilde{p}$, she is uncertain whether the good is supplied by the high quality or the low quality seller. She therefore wants the good inspected by buying certification with positive probability. Through inspection, the buyer tries to dissuade the low quality seller to set the "cheating" price $\tilde{p}$. Yet, as in an inspection game, the buyer has only an incentive to buy certification and inspect when the low quality seller cheats "often enough". This gives rise to the use of mixed strategies. As in an inspection game the buyer's certification probability is such that the low quality seller is indifferent between cheating, i.e., setting the high price $\tilde{p}$, and honestly signaling his low quality by setting the price $q_{l}$. On the other hand, the probability with which the low quality seller chooses the high price $\tilde{p}$ is such that the buyer is indifferent between buying the good uncertified and asking for certification.

Proposition 1 also describes the certifier's profits in the subgame $\Gamma\left(p_{c}\right)$. The certifier anticipates this outcome when choosing its price $p_{c}$ for certifying the good's quality. When the certifier maximizes its profits $\Pi_{c}$ with respect to the certification price $p_{c}$, it must take into account that $\tilde{\mu}$ depends on $p_{c}$ itself and the certifier therefore anticipates that the very case distinction $\lambda \lessgtr \tilde{\mu}$ and $c_{h} \gtrless \tilde{p}$ depends on its choice of $p_{c}$. The following proposition shows that expression (4) is increasing in $p_{c}$. Hence, the certifier picks the largest price such that $\lambda \leq \tilde{\mu}$ and $c_{h} \leq \tilde{p}$.

Proposition 2 Consider the full game with buyer-induced certification.

i.) Suppose that $\lambda \leq 1 / 2$ and $c_{h} \leq\left(q_{h}+q_{l}\right) / 2$. Then the certifier sets a price $p_{c}^{b}=\Delta q / 4$ and obtains a profit of

$$
\Pi_{c}^{b}=\frac{\lambda \Delta q}{2\left(q_{h}+q_{l}\right)}\left(\Delta q-4 c_{c}\right) .
$$

ii.) Suppose that $\lambda>1 / 2$ or $c_{h}>\left(q_{h}+q_{l}\right) / 2$. Then the certifier sets the price $p_{c}^{b}=\left(q_{h}-c_{h}\right)\left(c_{h}-q_{l}\right) / \Delta q$ and obtains a profit of

$$
\Pi_{c}^{b}=\frac{\lambda\left[\left(q_{h}-c_{h}\right)\left(c_{h}-q_{l}\right)-\Delta q c_{c}\right]}{c_{h}} .
$$

We argued that the monopolistic certifier benefits from a relatively high uncertainty for the buyer and an intermediate price of the good; we also 
showed that the buyer's willingness to pay for certification is maximized for $\hat{\mu}=1 / 2$ and $\hat{p}=\left(q_{h}+q_{l}\right) / 2$. A comparison demonstrates that, for the parameter constellation $\lambda \leq 1 / 2$ and $c_{h} \leq\left(q_{h}+q_{l}\right) / 2$, the equilibrium induces exactly this outcome. Indeed, the certifier's optimal price $p_{c}=\Delta q / 4$ leads to a price $p=\left(q_{h}+q_{l}\right) / 2$ and a belief $\mu=1 / 2$ and maximizes the expression

$$
\min \left\{\Delta U^{1}, \Delta U^{2}\right\}
$$

For $c_{h}>\left(q_{h}+q_{l}\right) / 2$, the price $p=\left(q_{h}+q_{l}\right) / 2$ would imply a loss to the high quality seller and, intuitively, the certifier cannot induce this maximum degree of uncertainty. For $\lambda>1 / 2$, the ex ante belief of the buyer about the product exceeds $1 / 2$. Consequently, the certifier is unable to induce the belief $\mu=1 / 2$. Instead, the certifier is restricted and maximizes the expression $\min \left\{\Delta U^{1}, \Delta U^{2}\right\}$ under a feasibility constraint. That is, the certifier's price maximizes the buyer's uncertainty about the seller's quality and, thereby, her willingness to pay.

\section{Seller Induced Certification}

In this section we consider the case where the seller instead of the buyer may buy certification. Here certification plays a different role. Rather than giving the buyer the possibility to protect herself from bad quality, it enables a high quality seller to ascertain the quality of his product to the buyer. Although the distinction seems small, it has a major impact on the equilibrium outcome, primarily because only the high quality seller is prepared to demand certification. Because of this, we can show that seller-induced certification is simpler and easier to control by the certifier.

Under seller-induced certification the parties play the following game:

$\mathrm{t}=1$ The certifier sets a price $p_{c}$.

$\mathrm{t}=2$ Nature selects the quality $q_{i}, i \in\{l, h\}$ of the good offered by the seller.

$\mathrm{t}=3$ The seller offering the good at quality $q_{i}$ and cost $c_{i}$ decides about the price $p$ at which he offers the good.

$\mathrm{t}=4$ The seller decides whether or not to demand certification for his good. 
$\mathrm{t}=5$ The buyer decides whether or not to buy the good.

Thus, in comparison to the model described in the previous section, we only change stage four by letting the seller, rather than the buyer, decide about certification. Note that the sequence of stages 3 and 4 is immaterial. Our setting where the seller first chooses his price and then decides about certification is strategically equivalent to the situation where he simultaneously takes both decisions, or reverses their order.

We again focus on Perfect Bayesian Equilibria of this game. Note again that after the certifier has set his price $p_{c}$ a proper subgame, $\Gamma\left(p_{c}\right)$, starts with nature's decision about the quality offered by the seller. The subgame $\Gamma\left(p_{c}\right)$ is a pure signalling game if the seller does not buy certification in stage 4. In contrast, if the seller does decide to certify, the quality is revealed to the buyer, and there is no asymmetric information. In the subsequent subgame, the $q_{h}$ seller sells his good at price $p=q_{h}$, whence the low quality seller sells his good at a price $p=q_{l}$.

In order to capture the seller's option to certify, we expand the actions open to the seller by an action $c$ that represents the seller's option to certify and to charge the maximum price $q_{i}$. Hence, the seller's payoff associated with the action $c$ are $\Pi_{h}(c)=q_{h}-c_{h}$ and $\Pi_{l}(c)=q_{l}$ for a high and low quality seller, respectively. Let $\sigma_{i}(c)$ denote the probability that the $q_{i}$ seller buys certification. We further adopt the notation of the previous section. Then we may express a mixed strategy of the seller $q_{i}$ over certification and a, possibly, infinite but countable number of prices by probabilities $\sigma_{i}\left(p_{j}\right)$ such that

$$
\sigma_{i}(c)+\sum_{j} \sigma_{i}\left(p_{j}\right)=1
$$

In contrast to the previous section, the buyer can no longer decide to buy certification so that her actions are now constrained to $s_{n n}$ and $s_{n b}$. As before let $\mu(p)$ represent the buyer's belief upon observing a non-certified good priced at $p$. Consequently, $s_{n b}$ is individually rational whenever

$$
\mu(p) \Delta q+q_{l} \geq p
$$


and $s_{n n}$ is individually rational whenever

$$
\mu(p) \Delta q+q_{l} \leq p
$$

Proposition 3 For any price of certification $p_{c}<q_{h}-c_{h}$, the equilibrium outcome in the subgame $\Gamma\left(p_{c}\right)$ is unique. The high quality seller certifies with probability 1 and obtains the profit $\Pi_{h}^{*}=q_{h}-c_{h}-p_{c}>0$, whereas the low quality seller does not certify and obtains the payoff $\Pi_{l}^{*}=q_{l}$. For any price $p_{c}>q_{h}-c_{h}$, any equilibrium outcome of the subgame $\Gamma\left(p_{c}\right)$ involves no certification. For $p_{c}=q_{h}-c_{h}$, the subgame $\Gamma\left(p_{c}\right)$ has an equilibrium in which high quality seller certifies with probability 1 and obtains the profit $\Pi_{h}^{*}=0$, whereas the low quality seller does not certify and obtains the payoff $\Pi_{l}^{*}=q_{l}$.

The proposition characterizes the equilibrium outcome of the subgame $\Gamma\left(p_{c}\right)$. From this characterization, we can derive the equilibrium of the overall game of seller-induced certification.

Proposition 4 The full game with seller-induced certification has the unique equilibrium outcome $p_{c}=q_{h}-c_{h}$ with equilibrium payoffs $\Pi_{c}^{s}=\lambda\left(q_{h}-c_{h}-c_{c}\right)$, $\Pi_{h}^{*}=0$, and $\Pi_{l}^{*}=q_{l}$.

Comparing the outcome of seller-induced certification with the outcome under buyer-induced certification we get the following result.

Proposition 5 The certifier obtains a higher profit under seller-induced than under buyer-induced certification: $\Pi_{c}^{s}>\Pi_{c}^{b}$.

The proposition shows that the certifier is better off when it sells certification to the seller. The intuition behind this result is that if the buyer decides whether or not to certify, the decision to certify cannot be made contingent on the actual quality. This is different from when the seller has the right to decide about certification. Clearly, a seller with low quality $q_{l}$ will never demand certification. In contrast, we showed that, in any equilibrium, the seller $q_{h}$ always certifies. The intuition is that if seller $q_{h}$ does not certify at a price $p_{c}$ quoted by the certifier, then the certifier gets zero profits from the seller. It, therefore, does strictly better by lowering the certification price to a level where it is worthwhile for the seller to demand certification. 


\section{Welfare}

Certification enables the high quality seller to sell his good. This obvious increase in social efficiency obtains both under buyer- and seller-induced certification. From an efficiency perspective, the differences between the two regimes relate to differences in the probability at which the low quality good is sold, and differences in the cases in which costly certification arises.

First, under seller-induced certification the low quality good is always sold, if offered at all. This is different under buyer-induced certification, where the good is not sold when the low quality seller picks the high price $\tilde{p}$ and the buyer certifies. This happens with probability

$$
\omega=\sigma_{l}^{*}(\tilde{p}) \sigma^{*}\left(s_{c h} \mid \tilde{p}, \mu^{*}(\tilde{p})\right) .
$$

Thus, under buyer-induced certification an efficiency loss of $q_{l}$ occurs with probability $(1-\lambda) \omega$.

Second, the different regimes may lead to different intensities of certification and therefore differences in expected certification costs. In particular, the probability of certification under buyer-induced certification is

$$
x^{b}=\left[\lambda+(1-\lambda) \sigma_{l}^{*}(\tilde{p})\right] \sigma\left(s_{c h} \mid \tilde{p}, \mu^{*}(\tilde{p})\right) .
$$

Remember that the buyer demands certification only if the seller quotes a high price. Now, the cornered bracket contains the probabilities at which the seller quotes that high price, which include the probability $\lambda$ at which he sells the high quality product, and the probability $(1-\lambda) \sigma_{l}^{*}(\tilde{p})$ by which he has a low quality product but quotes the high price.

By comparison, under seller-induced certification the probability of certification is

$$
x^{s}=\lambda .
$$

Let $W F^{i}, i=b, s$ denote social welfare under buyer and seller-induced certification, respectively. As usual, it is defined as the sum of consumer and producer surplus. Then, social welfare under buyer-induced certification is

$$
W F^{b}=\lambda\left(q_{h}-c_{h}\right)+(1-\lambda)(1-\omega) q_{l}-x^{b} c_{c},
$$


whereas social welfare under seller-induced certification equals

$$
W F^{s}=\lambda\left(q_{h}-c_{h}\right)+(1-\lambda) q_{l}-x^{s} c_{c} .
$$

Consequently, the difference in social welfare between the two regimes is

$$
\Delta W F=W F^{s}-W F^{b}=(1-\lambda) \omega q_{l}+\left(x^{b}-x^{s}\right) c_{c},
$$

In Proposition 5 we have established that the profits of a monopolistic certifier are larger under seller certification. The certifier will therefore have a preference for seller-induced certification. We now check whether these preferences are aligned with social efficiency. Clearly, when certification costs are zero, this follows immediately. The more interesting case is therefore when the cost of certification, $c_{c}$, is strictly positive. In this case, the certifier's preferences are still in line with social efficiency, when the probability of certification is smaller under seller-induced certification. In the next lemma we compare the probabilities of certification in both regimes.

Lemma 5 For $\lambda>1 / 2$ or $c_{h}>\left(q_{h}+q_{l}\right) / 2$ the probability of certification under seller-induced certification, $x^{s}$, is lower than under buyer-induced certification, $x^{b}$. For $\lambda \leq 1 / 2$ and $c_{h} \leq\left(q_{h}+q_{l}\right) / 2$ the probability of certification under seller-induced certification, $x^{s}$, is higher than under buyer-induced certification, $x^{b}$, if and only if $q_{h}<3 q_{l}$.

The lemma identifies a case where the probability of certification is higher under seller-induced certification than under buyer-induced certification. This leaves open the possibility that the decision of a monopolistic certifier to offer its services to the seller rather than the buyer is not in the interest of social efficiency. In particular, if certification costs, $c_{c}$, are large, the certifier's decision may be suboptimal. Yet, the following proposition shows that this possibility does not arise. Whenever the certifier's profit under buyer-induced certification is non-negative, social welfare is larger under seller-induced certification, in spite of possibly a higher probability of certification.

Proposition 6 Social welfare is higher under seller-induced certification than under buyer-induced certification. 


\section{$6 \quad$ Extensions}

Our central result that the certifier is better off selling its services to the better informed party, and that its decision is socially efficient is derived within a very stylized model. In this section, we informally discuss extensions in order to show that our result is robust.

To begin, we assumed that, because of the high price, the buyer does not purchase the good in spite of gains from trade, if certification reveals low quality. This assumption is realistic as long as the costs of renegotiating the price after certification are sufficiently high. Yet our results do not depend on the absence of renegotiation. To see this, suppose renegotiation is costless so that, after certification, the buyer and a low quality seller always renegotiate to trade the low quality good at the price $p=q_{l}$. In this case, the low quality seller always has an incentive to quote the higher price for the low quality good before certification, because he is ensured the low quality price even when the buyer demands certification. Hence, ex post renegotiation actually worsens the outcome of the inspection game by raising the seller's cheating incentives - yet it does not change the outcome of the signalling game.

Our results are also robust to the introduction of imperfect certification technologies. Consider a certification technology that reveals the correct quality only with probability $\pi>1 / 2$, whereas it identifies the wrong quality with probability $1-\pi$. Although the imperfect certification technology reduces the profitability of buyer-induced certification, it does not qualitatively change the equilibrium. Intuitively, a less informative certification technology shrinks the intermediate area in Figure 1, where $S\left(s_{c h}\right)$ is optimal, in a continuous way. Imperfect certification also does not change the nature of the equilibrium outcome with seller-induced certification. In particular, an equilibrium exists where the certifier charges the certification price $p_{c}=\pi q_{h}-c_{h}$, the high quality seller certifies and charges the price $q_{h}$, and the low quality seller sells the good uncertified at a price $q_{l}$. The equilibrium is sustained by a buyer who buys the good at the price $q_{h}$ only if it is certified as of high quality and, consistent with equilibrium play, only believes that the good has high quality when it is certified and the price is $q_{h}$. Hence, as shown in Strausz (2010) and in contrast to De and Nabar (1991), the equilibrium 
outcome remains separating also with imperfect certification. Consequently, the equilibrium outcomes under buyer- and seller-induced certification are continuous in $\pi$. As a result, our results are robust to imperfect certification technologies that are not completely uninformative.

Starting from an industrial organization perspective, we assumed that the buyer, seller, and certifier can only use unconditional prices rather than sophisticated contracts to coordinate their exchange. This raises the question whether more complicated contracts, such as prices that condition on the certification outcome, can change our ranking between seller-induced and buyer-induced certification. As one can formally show with optimal mechanism design, this is not the case. The intuition is that with seller-induced certification, the certifier extracts all the rents from certification, and hence, the certifier cannot do better than in our context with seller-induced certification. Stated more formally, the equilibrium payoffs under the optimal mechanism coincide with the equilibrium payoffs in our certification game with seller-induced certification.

In the baseline model, the seller can produce only one fixed quality. Suppose alternatively that a high quality producer actually has the choice to produce alternatively high or low quality, whence a low quality producer can produce only low quality. In this case, the high quality seller's next best alternative to producing high quality and having this certified is to sell low quality without certification. This changes the outside option of the high quality seller from zero to $q_{l}$ and limits the certifier's possibility to exploit him. Nevertheless, all our qualitative results are upheld. In particular, the certifier obtains the higher profits from seller-induced certification, because, as explained in the previous paragraph, it enables it to extract all rents from certification - even though the rents from certification are now smaller. Similarly, welfare is higher under seller-induced certification.

We finally emphasize that the bilateral seller-buyer framework, within which we have developed our argument, is not crucial. As a particular example, consider a setting which applies particularly well to the financial market, where one seller can sell $n$ units of the good to $n$ identical buyers. Essentially, there are two possible information structures. A first one in which 
buyers cannot share the certification result but each individually must buy certification. Under buyer certification, our formal results carry through and, hence, the certifier's profits are simply multiplied by $n$. Under seller certification, Proposition 3 is changed so that the profits from selling the product are also multiplied by $n$, and $p_{c}=n\left[q_{h}-c_{h}\right]$. Because the certifier's profits from selling to buyers and sellers are both multiplied by $n$, both the ranking of seller-induced vs. buyer-induced certification by the certifier and from a welfare point of view are as in our baseline model.

The second information structure is one in which buyers collude to collectively initiate certification. Under buyer certification, the market structure remains as in the baseline model, yet with $n$ times the buyer's benefit that can be exploited by the certifier. Under seller certification, the same change of Proposition 3 takes place as above. Again, the results remain unchanged.

\section{$7 \quad$ Empirical Examples}

Our model and results apply one-to-one to situations in which certification is both product and customer specific. This is the case, for example, in the automotive industry. We first argue that this industry motivates particularly well our theoretical model used. ${ }^{6}$ We then move on to other examples - in particular to certification in the financial market.

In the automotive industry, most of the development and production of a complex part for a premium automobile is done by only one supplier - the seller, whom the automotive producer - the buyer — selects explicitly. Because the part is customer specific, the buyer-seller relationship is a bilateral monopoly. Moreover, before the so called null-series production, information between the buyer and the seller about the quality of the part is asymmetric. The automotive industry provides independent certifiers, whose role is

\footnotetext{
${ }^{6}$ The evidence is taken from Mueller et al. (2008), and from a large scale study conducted in 2007/08 by Konrad Stahl et al. for the German Association of Automotive Manufacturers (VDA) on Upstream Relationships in the Automotive Industry. Survey participants were car producers and their upstream suppliers. All German car producers and 13 first tier counterparts were questioned as to their procurement relationships.
} 
to mediate these information asymmetries. ${ }^{7}$ Due to significant economies of scope involving the analytical instruments, the certification industry is highly concentrated. One of the key test criteria is the fulfilment of safety norms. It turns out that the testing of car modules and systems is predominantly performed on the request of the upstream supplier rather than the buyer. Moreover, the buyer conditions his actual purchase on the quality certification. Our model, therefore, captures the procurement relationships in the automotive industry and our results are consistent with the observations in this industry.

While our model applies particularly well to cases in which certification is both product and customer specific, the results also help us understanding purely product specific certification. Examples of purely product specific certification range from the certification of foodstuff for production without herbicides or pesticides; to the certification of toys for production without aggressive chemicals, to the certification of building materials, or of fire-proof safes.

A particularly timely and controversially discussed example is certification in the financial industry. Before the financial crises was triggered, financial products were certified by a heavily concentrated rating industry. The fact that many actual buyers now admit that they poorly understood the products' complexities underscores the large degree of asymmetric information in this market and the rating agencies' central role in reducing it. Before the crisis and consistent with our result, certification was initiated by the issuers - the sellers, who paid rating agencies. A controversial claim is that seller-induced certification led to capture of the certifier and inflated ratings, which precipitated the financial crisis. Proponents of this claim, therefore, argue for a regulatory response to transfer the rating decision from sellers to buyers.

Yet by our results, certification should continue to be initiated by the

\footnotetext{
${ }^{7}$ An example is EDAG, an engineering company centering on the development and prototype-construction of cars, as well as on independent certification of car modules and systems. In this function it serves all major car producers world wide. See http://www.edag.de/produkte/prueftechnik/automotive/index_html
} 
sellers, since this has robust welfare superior properties. Given these welfare properties, we caution against regulatory pressure in favor of buyerrather than seller-induced certification. Since capture is the issue, regulatory initiative should concentrate on directly preventing this, by designing a certification system in which capture is minimized or excluded. A particularly successful example of this is the German "Stiftung Warentest" originally founded by the Federal Government to prevent capture, and later privatized. Yet the design of an efficient, capture-proof regulatory mechanism addressing certification in financial markets lies beyond the scope of this paper.

\section{Conclusion}

Under asymmetric quality information, a demand for certification may arise from both buyers and sellers. Buyers do not want to be cheated if offered a good of unknown quality at a high price. In turn, sellers want to offer the good at a high price - especially if it is of high quality. So to whom does, and, from a welfare point of view, to whom should a credible certifier sell his services, to the buyer or to the seller? Within a parsimonious model, we give straightforward answers to these questions: a certifier does, and should sell to the better informed party.

While the answers to these questions appear deceptively simple, their justification needs an elaborate argument. In particular, we show that certification to the buyer and certification to the seller play very different economic roles and lead to different games, namely an inspection game with the typical mixed strategy equilibrium (which is semi-separating in our context), and a signalling game with a separating equilibrium, respectively.

Our result is consistent with certification in real life - in particular in the automobile industry and financial markets. As to the current discussion about certification in the latter markets, it leads to a clear policy implication. In contrast to much of the current discussion about transferring the initiation to certify to buyers, we provide an argument in favor of seller-initiated certification. This may caution policy makers to think of other means to prevent capture rather than simply reverting from seller- to buyer-induced certification. 
We also demonstrated the robustness of our results by considering many extensions. Clearly, further extensions and refinements of the approach are possible. In order to focus on our central point, we have purposively excluded seller reactions to certification, such as adapting quality, as this is discussed in other papers. For the same reason, we also have excluded certifier capture by the seller. Finally, we excluded competition between many sellers, or many certifiers. Arguably, the latter is less important, in view of the technical economies of scale and reputation effects associated with certification. The former, competition between sellers, enhances sellers's demand for certification, but tends not to qualitatively change our insights.

\section{Appendix}

The appendix contains all formal proofs to our Lemmata and Propositions.

Proof of Lemma 1: Follows directly from the text.

Q.E.D.

Proof of Lemma 2: To show that $\Pi_{h}\left(p, \mu \mid \sigma^{*}\right)$ is non-decreasing in $\mu$ we first establish that, in any $\mathrm{PBE}, \sigma^{*}\left(s_{n n} \mid p, \mu\right)$ is weakly decreasing in $\mu$. Suppose not, then we may find $\mu_{1}<\mu_{2}$ such that $0 \leq \sigma^{*}\left(s_{n n} \mid p, \mu_{1}\right)<$ $\sigma^{*}\left(s_{n n} \mid p, \mu_{2}\right) \leq 1$. Lemma 1 implies that $\left(p, \mu_{2}\right) \in S\left(s_{n n} \mid p_{c}\right)$. That is,

$$
p \geq \mu_{2} q_{h}+\left(1-\mu_{2}\right) q_{l}
$$

and

$$
p_{c} \geq \mu_{2}\left(q_{h}-p\right)
$$

Now since $\sigma^{*}\left(s_{n n} \mid p, \mu_{1}\right)<1$ we have either $\sigma^{*}\left(s_{n b} \mid p, \mu_{1}\right)>0$ or $\sigma^{*}\left(s_{c h} \mid p, \mu_{1}\right)>$ 0 . Suppose first $\sigma^{*}\left(s_{n b} \mid p, \mu_{1}\right)>0$, then by Lemma 1 we have $p \leq \mu_{1} q_{h}+(1-$ $\left.\mu_{1}\right) q_{l}$. But from $\mu_{2}>\mu_{1}$ and $q_{h}>q_{l}$ it then follows that $\mu_{2} q_{h}+\left(1-\mu_{2}\right) q_{l}>p$, which contradicts (6). Suppose therefore that $\sigma^{*}\left(s_{c h} \mid p, \mu_{1}\right)>0$, then by Lemma 1 we have $\mu_{1}\left(q_{h}-p\right) \geq p_{c}>0$. This requires $q_{h}>p$. But then, due to $\mu_{2}>\mu_{1}$, we get $\mu_{2}\left(q_{h}-p\right)>p_{c}$, which contradicts $(7)$.

Hence, we establish that $\sigma^{*}\left(s_{n n} \mid p, \mu\right)$ is weakly decreasing in $\mu$ and therefore $\sigma^{*}\left(s_{n b} \mid p, \mu\right)+\sigma^{*}\left(s_{c h} \mid p, \mu\right)$ must be weakly increasing in $\mu$. Consequently, $\Pi_{h}\left(p, \mu \mid \sigma^{*}\right)$ is weakly increasing in $\mu$. 
Next we show that in any $\operatorname{PBE} \sigma^{*}\left(s_{n b} \mid p, \mu\right)$ is weakly increasing in $\mu$. Suppose not, then we may find $\mu_{1}<\mu_{2}$ such that $1 \geq \sigma^{*}\left(s_{n b} \mid p, \mu_{1}\right)>$ $\sigma^{*}\left(s_{n b} \mid p, \mu_{2}\right) \geq 0$. Since $\sigma^{*}\left(s_{n b} \mid p, \mu_{1}\right)>0$, Lemma 1 implies that $\left(p, \mu_{1}\right) \in$ $S\left(s_{n b} \mid p_{c}\right)$. That is,

$$
p \leq \mu_{1} q_{h}+\left(1-\mu_{1}\right) q_{l}
$$

and

$$
p_{c} \geq\left(1-\mu_{1}\right)\left(p-q_{l}\right) \text {. }
$$

Now since $\sigma^{*}\left(s_{n b} \mid p, \mu_{2}\right)<1$ we have either $\sigma^{*}\left(s_{n n} \mid p, \mu_{2}\right)>0$ or $\sigma^{*}\left(s_{c h} \mid p, \mu_{2}\right)>$ 0 . Suppose first $\sigma^{*}\left(s_{n n} \mid p, \mu_{2}\right)>0$, then by Lemma 1 this implies $p \geq$ $\mu_{2} q_{h}+\left(1-\mu_{2}\right) q_{l}$. But due to $\mu_{2}>\mu_{1}$ and $q_{h}>q_{l}$ we get $p>\mu_{1} q_{h}+\left(1-\mu_{1}\right) q_{l}$. This contradicts (8). Suppose therefore that $\sigma^{*}\left(s_{c h} \mid p, \mu_{2}\right)>0$, then by Lemma 1 we have $\left(1-\mu_{2}\right)\left(p-q_{l}\right) \geq p_{c}>0$. This requires $p>q_{l}$. But then, due to $\mu_{2}>\mu_{1}$, we get $\left(1-\mu_{1}\right)\left(p-q_{l}\right)>p_{c}$. This contradicts (9). Hence, $\sigma^{*}\left(s_{n b} \mid p, \mu\right)$ must be weakly increasing in $\mu$. Consequently, $\Pi_{l}\left(p, \mu \mid \sigma^{*}\right)$ is weakly increasing in $\mu$.

Q.E.D.

Proof of Lemma 3: i) For any $\bar{p}<q_{l}, \mu \in[0,1]$ we have $(\bar{p}, \mu) \notin S\left(s_{n n}\right)$, $(\bar{p}, \mu) \notin S\left(s_{c h}\right)$ and $(\bar{p}, \mu) \in S\left(s_{n b}\right)$. Hence, $\sigma^{*}\left(s_{n b} \mid \bar{p}, \mu^{*}(\bar{p})\right)=1$. Now suppose for some $\bar{p}<q_{l}$ we have $\sigma_{i}^{*}(\bar{p})>0$. This would violate (2), because instead of charging $\bar{p}$ seller $q_{i}$ could have raised profits by $\varepsilon \sigma_{i}(\bar{p})$ by charging the higher price $\bar{p}+\varepsilon<q_{l}$ with $\varepsilon \in\left(0,\left(q_{l}-\bar{p}\right)\right)$. At $\bar{p}+\varepsilon<q_{l}$ the buyer always buys, because, as established, $\sigma^{*}\left(s_{n b} \mid \bar{p}+\varepsilon, \mu\right)=1$ for all $\mu$ and in particular for $\mu=\mu^{*}(\bar{p}+\varepsilon)$.

For any $\bar{p}>q_{h}, \mu \in[0,1]$ we have $(\bar{p}, \mu) \in S\left(s_{n n}\right),(\bar{p}, \mu) \notin S\left(s_{c h}\right)$ and $(\bar{p}, \mu) \notin S\left(s_{n b}\right)$. Hence, $\sigma^{*}\left(s_{n n} \mid \bar{p}, \mu^{*}(\bar{p})\right)=1$. Now suppose we have $\sigma_{l}(\bar{p})>0$. This would violate $(2)$, because instead of charging $\bar{p}$ seller $q_{l}$ could have raised profits by $\left(q_{l}-\varepsilon\right) \sigma_{l}(\bar{p})$ by charging the price $q_{l}-\varepsilon$.

ii) Suppose $q_{l}-\Pi_{l}^{*}=\delta>0$. Now consider a price $p^{\prime}=q_{l}-\varepsilon$ with $\varepsilon \in(0, \delta)$ then for any $\mu^{\prime} \in[0,1]$ we have $\left(p^{\prime}, \mu^{\prime}\right) \in S\left(s_{n b}\right)$ and $\left(p^{\prime}, \mu^{\prime}\right) \notin$ $S\left(s_{n n}\right) \cup S\left(s_{c h}\right)$ so that, by $(1)$, we have $\sigma^{*}\left(s_{n b} \mid p^{*}, \mu^{*}\left(p^{\prime}\right)\right)=1$ and, therefore, $\Pi_{l}\left(p^{*}, \mu^{*}\left(p^{\prime}\right) \mid \sigma^{*}\right)=p^{\prime}>\Pi_{l}^{*}$. This contradicts $(2)$.

iii) For any $p$ such that $\sigma_{h}^{*}(p)>0$, we have $\Pi_{h}^{*}=\Pi_{h}\left(p, \mu^{*}(p) \mid \sigma^{*}\right)=$ $\left[\sigma^{*}\left(s_{n b} \mid p, \mu^{*}(p)\right)+\sigma^{*}\left(s_{c h} \mid p, \mu^{*}(p)\right)\right] p-c_{h}$. As argued in i), we have $\sigma^{*}\left(s_{n n} \mid p, \mu\right)=$ 
1 for all $p>q_{h}$ and $\mu \in[0,1]$. Hence, $\Pi_{h}\left(p, \mu \mid \sigma^{*}\right)=0$ whenever $p>q_{h}$. But for any price $p \leq q_{h}$ we have $\Pi_{h}\left(p, \mu \mid \sigma^{*}\right) \leq q_{h}-c_{h}$. Hence, it follows that $\Pi_{h}^{*} \leq q_{h}-c_{h}$. Now suppose $\Pi_{h}^{*}=q_{h}-c_{h}$. Then we must have $\sigma_{h}^{*}\left(q_{h}\right)=1$ and $\sigma^{*}\left(s_{n b} \mid q_{h}, \mu^{*}\left(q_{h}\right)\right)+\sigma^{*}\left(s_{c h} \mid q_{h}, \mu^{*}\left(q_{h}\right)\right)=1$. But, due to $\mu^{*}\left(q_{h}\right)\left(q_{h}-q_{h}\right)=0<p_{c}$, we have $\left(q_{h}, \mu^{*}\left(q_{h}\right)\right) \notin S\left(s_{c h} \mid q_{h}\right)$ so that $\sigma^{*}\left(s_{c h} \mid q_{h}, \mu^{*}\left(q_{h}\right)\right)=0$. Hence, we must have $\sigma^{*}\left(s_{n b} \mid q_{h}, \mu^{*}\left(q_{h}\right)\right)=1$. This requires $\left(q_{h}, \mu^{*}\left(q_{h}\right)\right) \in S\left(s_{n b} \mid p_{c}\right)$ so that we must have $\mu^{*}\left(q_{h}\right)=1$. By (3), this requires $\sigma_{l}^{*}\left(q_{h}\right)=0$. But since $\Pi_{l}\left(q_{h}, 1 \mid \sigma^{*}\right)=\sigma^{*}\left(s_{n b} \mid q_{h}, \mu^{*}\left(q_{h}\right)\right) q_{h}=q_{h}$ we must, by (2), have $\Pi_{l}^{*} \geq q_{h}$. Together with $\sigma_{l}^{*}\left(q_{h}\right)=0$, it would require $\sigma_{l}^{*}(p)>0$ for some $p>q_{h}$ and leads to a contradiction with i). Q.E.D.

Proof of Lemma 4: We first prove ii): Suppose to the contrary that $\delta \equiv \tilde{p}-c_{h}-\Pi_{h}^{*}>0$. Then, due to the countable number of equilibrium prices, we can find an out-of-equilibrium price $p^{\prime}=\tilde{p}-\varepsilon$ for some $\varepsilon \in(0, \delta)$. Then for any belief $\mu^{\prime} \in\left(p_{c} /\left(q_{h}-p^{\prime}\right), 1-p_{c} /\left(p^{\prime}-q_{l}\right)\right) \neq \emptyset^{8}$ we have $\left(p^{\prime}, \mu^{\prime}\right) \in S\left(\sigma_{c h}\right)$ and $\left(p^{\prime}, \mu^{\prime}\right) \notin S\left(\sigma_{n n}\right) \cup S\left(\sigma_{n b}\right)$. Consequently, $\sigma^{*}\left(s_{c h} \mid p^{\prime}, \mu^{\prime}\right)=1$. Hence, $\Pi_{h}\left(p^{\prime}, \mu^{\prime}\right)=p^{\prime}-c_{h}=\tilde{p}-c_{h}-\varepsilon>\tilde{p}-c_{h}-\delta=\Pi_{h}^{*}$ and $\Pi_{l}\left(p^{\prime}, \mu^{\prime}\right)=0<q_{l} \leq \Pi_{l}^{*}$. Therefore, by B.R. the buyer's equilibrium belief must satisfy $\mu^{*}\left(p^{\prime}\right) \geq \mu^{\prime}$. By Lemma 2 it follows $\Pi_{h}\left(p^{\prime}, \mu^{*}\left(p^{\prime}\right)\right) \geq \Pi_{h}\left(p^{\prime}, \mu^{\prime}\right)=\tilde{p}-c_{h}-\varepsilon>\Pi_{h}^{*}$. This contradicts (2). Consequently, we must have $\Pi_{h}^{*} \geq \tilde{p}-c_{h}$. To show i) note that for all $p<\tilde{p}$ and $\mu \in[0,1]$ we have $\Pi_{h}(p, \mu \mid \sigma) \leq p-c_{h}<\tilde{p}-c_{h} \leq \Pi_{h}^{*}$ so that $\sigma_{h}(p)>0$ would violate $(2)$.

Q.E.D.

Proof of Proposition 1: i): First we show that for $\lambda<\tilde{\mu}$ and $c_{h}<\tilde{p}$ there exists no pooling, i.e., there exists no price $\bar{p}$ such that $\sigma_{h}^{*}(\bar{p})=\sigma_{l}^{*}(\bar{p})>$ 0 . For suppose there does. Then, by Lemma 4.i, we have $\bar{p} \geq \tilde{p}$ and, by Lemma 3.i, we have $\bar{p} \leq q_{h}$. Yet, due to (3) we have $\mu^{*}(\bar{p})=\lambda<\tilde{\mu}$ so that $q_{l}+\mu^{*}(\bar{p}) \Delta q-\bar{p}<q_{l}+\tilde{\mu} \Delta q-\tilde{p}=0$. Moreover, $\mu^{*}(\bar{p})\left(q_{h}-\bar{p}\right)<\tilde{\mu}\left(q_{h}-\tilde{p}\right)=p_{c}$. Therefore, $\sigma^{*}\left(s_{n n} \mid \bar{p}, \mu^{*}(\bar{p})\right)=1$ and $\Pi_{h}\left(\bar{p}, \mu^{*}(\bar{p})\right)=0$. As a result, $\sigma_{h}^{*}(\bar{p})>0$ contradicts (2), because, by Lemma $4 . \mathrm{ii}, \Pi_{h}^{*} \geq \tilde{p}-c_{h}>0=\Pi_{h}\left(\bar{p}, \mu^{*}(\bar{p})\right)$.

Second, suppose that for some $\bar{p}>\tilde{p}$ we have $\sigma_{h}^{*}(\bar{p})>0$ then, by definition of $\tilde{p}$, we have $(\bar{p}, \mu) \notin S\left(s_{c h}\right)$ for any $\mu \in[0,1]$. Hence, $\sigma^{*}\left(s_{c h} \mid \bar{p}, \mu^{*}(\bar{p})\right)=0$

\footnotetext{
${ }^{8}$ Let $l(p) \equiv p_{c} /\left(q_{h}-p\right)$ and $h(p) \equiv 1-p_{c} /\left(p-q_{l}\right)$. Then by the definition of $\tilde{p}$ we have $l(\tilde{p})=h(\tilde{p})$. Moreover, for $q_{l}<p<q_{h}$ we have $l^{\prime}(p)=p_{c} /\left(q_{h}-p\right)^{2}>h^{\prime}(p)=$ $p_{c} /\left(p-q_{l}\right)^{2}>0$. Hence, $l(\tilde{p}-\varepsilon)<h(\tilde{p}-\varepsilon)$ for $\varepsilon>0$ so that $\tilde{p}-\varepsilon>q_{l}$ and, therefore, $l\left(p^{\prime}\right)<h\left(p^{\prime}\right)$.
} 
so that $\Pi_{l}\left(\bar{p}, \mu^{*}(\bar{p})\right)=\Pi_{h}\left(\bar{p}, \mu^{*}(\bar{p})\right)+c_{h}$. From Lemma 4.ii it then follows $\Pi_{l}\left(\bar{p}, \mu^{*}(\bar{p})\right) \geq \tilde{p}$ and, therefore, $\sum_{p \geq \tilde{p}} \sigma_{l}^{*}(p)=1$. From $\bar{p}>\tilde{p}$ and $\tilde{\mu}>\lambda$ it follows $\lambda \Delta q+q_{l}-\bar{p}<\tilde{\mu} \Delta q+q_{l}-\tilde{p}=0$ so that $\lambda \Delta q+q_{l}<\bar{p}$. Now take a $\bar{p}>\tilde{p}$ with $\sigma_{l}(\bar{p})>0$ then, by Lemma 3 .ii and $(2), 0<q_{l} \leq \Pi_{l}^{*}=\Pi_{l}\left(\bar{p}, \mu^{*}(\bar{p}) \mid \sigma^{*}\right)=$ $\sigma\left(s_{n b} \mid \bar{p}, \mu^{*}(\bar{p})\right) \bar{p}$. This requires $\sigma\left(s_{n b} \mid \bar{p}, \mu^{*}(\bar{p})\right)>0$ and therefore $\left(\bar{p}, \mu^{*}(\bar{p})\right) \in$ $S\left(s_{n b} \mid p_{c}\right)$ and, hence, $\mu^{*}(\bar{p}) \Delta q+q_{l} \geq \bar{p}$. Combining the latter inequality with our observation that $\lambda \Delta q+q_{l}<\bar{p}$ and using (3), it follows

$$
\lambda \Delta q+q_{l}<\frac{\lambda \sigma_{h}^{*}(\bar{p})}{\lambda \sigma_{h}^{*}(\bar{p})+(1-\lambda) \sigma_{l}^{*}(\bar{p})} \Delta q+q_{l},
$$

which is equivalent to $\sigma_{h}^{*}(\bar{p})>\sigma_{l}^{*}(\bar{p})$. Summing over all $p \geq \tilde{p}$ and using $\sum_{p \geq \tilde{p}} \sigma_{l}^{*}(p)=1$ yields the contradiction $\sum_{p \geq \tilde{p}} \sigma_{h}^{*}(p)>1$. Hence, we must have $\sigma_{l}^{*}(\bar{p})=0$ for any $\bar{p}>\tilde{p}$. But this contradicts $\sum_{p \geq \tilde{p}} \sigma_{l}^{*}(p)=1$ and, therefore, we must have $\sigma_{h}^{*}(\bar{p})=0$ for all $\bar{p}>\tilde{p}$. Hence, if an equilibrium for $\lambda<\tilde{\mu}$ and $\tilde{p}>c_{h}$ exists then, by Lemma 4, it exhibits $\sigma_{h}^{*}(\tilde{p})=1, \Pi_{h}^{*}=\tilde{p}-c_{h}$ and $\sigma^{*}\left(s_{c h} \mid \tilde{p}, \tilde{\mu}\right)+\sigma^{*}\left(s_{n b} \mid \tilde{p}, \tilde{\mu}\right)=1$.

We now show existence of such an equilibrium and demonstrate that any such equilibrium has a unique equilibrium outcome. If $\sigma_{h}^{*}(\tilde{p})=1$ then (3) implies that $\mu^{*}(\tilde{p})=\tilde{\mu}$ whenever

$$
\sigma_{l}^{*}(\tilde{p})=\frac{\lambda(1-\tilde{\mu})}{\tilde{\mu}(1-\lambda)}
$$

which is smaller than one exactly when $\lambda<\tilde{\mu}$. By definition, $(\tilde{p}, \tilde{\mu}) \in S\left(s_{c h}\right) \cap$ $S\left(s_{n b}\right)$ so that any buying behavior with $\sigma^{*}\left(s_{c h} \mid \tilde{p}, \tilde{\mu}\right)+\sigma^{*}\left(s_{n b} \mid \tilde{p}, \tilde{\mu}\right)=1$ is consistent in equilibrium. In particular, $\sigma^{*}\left(s_{n b} \mid \tilde{p}, \tilde{\mu}\right)=q_{l} / \tilde{p}<1$ is consistent in equilibrium. Only for this buying behavior we have $\Pi_{l}\left(q_{l}, 0\right)=q_{l}=\Pi_{l}(\tilde{p}, \tilde{\mu})$ so that seller $q_{l}$ is indifferent between price $\tilde{p}$ and $q_{l}$. The equilibrium therefore prescribes $\sigma_{l}^{*}\left(q_{l}\right)=1-\sigma_{l}^{*}(\tilde{p})$. Finally, let $\mu^{*}\left(q_{l}\right)=0$ and $\sigma^{*}\left(s_{n b} \mid q_{l}, \mu^{*}\left(q_{l}\right)\right)=1$ and $\mu^{*}(p)=0$ for any price $p$ larger than $q_{l}$ and unequal to $\tilde{p}$. This out-ofequilibrium beliefs satisfies B.R.. Hence, the expected profit to the certifier is

$$
\Pi_{c}\left(p_{c}\right)=\left(\lambda+(1-\lambda) \sigma_{l}^{*}(\tilde{p})\right) \sigma^{*}\left(s_{c h} \mid \tilde{p}, \tilde{\mu}\right)\left(p_{c}-c_{c}\right)=\frac{\lambda\left(\tilde{p}-q_{l}\right)}{\tilde{\mu} \tilde{p}}\left(p_{c}-c_{c}\right) .
$$

ii) In order to show that, in any equilibrium of $\Gamma\left(p_{c}\right)$, we have $\Pi_{c}\left(p_{c}\right)=0$ whenever $\lambda>\tilde{\mu}$, we prove that for any $\bar{p}$ such that $\sigma^{*}\left(s_{c h} \mid \bar{p}, \mu^{*}(\bar{p})\right)>0$, it 
must hold $\sigma_{h}^{*}(\bar{p})=\sigma_{l}^{*}(\bar{p})=0$. Suppose we have $\sigma^{*}\left(s_{c h} \mid \bar{p}, \mu^{*}(\bar{p})\right)>0$, then $\left(\bar{p}, \mu^{*}(\bar{p})\right) \in S\left(s_{c h}\right)$ and, necessarily, $\bar{p} \leq \tilde{p}$. But by Lemma $4 . \mathrm{i}, \sigma_{h}^{*}(\bar{p})>0$ also implies $\bar{p} \geq \tilde{p}$. Therefore, we must have $\bar{p}=\tilde{p}$. But $(\tilde{p}, \mu) \in S\left(s_{c h}\right)$ only if $\mu=\tilde{\mu}$. Hence, we must have $\mu^{*}(\tilde{p})=\tilde{\mu}$. By (3) it therefore must hold

$$
\tilde{\mu}=\mu^{*}(\tilde{p})=\frac{\lambda \sigma_{h}^{*}(\tilde{p})}{\lambda \sigma_{h}^{*}(\tilde{p})+(1-\lambda) \sigma_{l}^{*}(\tilde{p})} .
$$

For $\lambda>\tilde{\mu}$ this requires $\sigma_{h}^{*}(\tilde{p})<\sigma_{l}^{*}(\tilde{p}) \leq 1$ and therefore there is some other $p^{\prime}>\tilde{p}$ such that $\sigma_{h}^{*}\left(p^{\prime}\right)>0$. But if also $p^{\prime}$ is an equilibrium price, then $\Pi_{h}\left(\tilde{p}, \mu^{*}(\tilde{p}) \mid \sigma^{*}\right)=\Pi_{h}\left(p^{\prime}, \mu^{*}\left(p^{\prime}\right) \mid \sigma^{*}\right)$. Yet, for any $p^{\prime}>\tilde{p}$ it holds $\left(p^{\prime}, \mu\right) \notin$ $S\left(s_{c h} \mid p_{c}\right)$ for any $\mu \in[0,1]$ so that $\Pi_{l}\left(p^{\prime}, \mu \mid \sigma^{*}\right)=\Pi_{h}\left(p^{\prime}, \mu \mid \sigma^{*}\right)+c_{h}$ and, together with our assumption $\sigma^{*}\left(s_{c h} \mid \bar{p}, \mu^{*}(\bar{p})\right)>0$ yields $\Pi_{l}\left(\bar{p}, \mu^{*}(\bar{p}) \mid \sigma^{*}\right)<$ $\Pi_{h}\left(\bar{p}, \mu^{*}(\bar{p}) \mid \sigma^{*}\right)+c_{h}=\Pi_{h}\left(p^{\prime}, \mu^{*}\left(p^{\prime}\right) \mid \sigma^{*}\right)+c_{h}=\Pi_{l}\left(p^{\prime}, \mu^{*}\left(p^{\prime}\right) \mid \sigma^{*}\right)$ so that, by (2), $\sigma_{l}^{*}(\bar{p})=0$. Since $\bar{p}=\tilde{p}$, this violates $\sigma_{l}^{*}(\tilde{p})>\sigma_{h}^{*}(\tilde{p}) \geq 0$. As a result, $\sigma^{*}\left(s_{c h} \mid \bar{p}, \mu^{*}(\bar{p})\right)>0$ implies $\sigma_{h}^{*}(\bar{p})=0$.

In order to show that we must also have $\sigma_{l}^{*}(\bar{p})=0$, assume again that $\sigma^{*}\left(s_{c h} \mid \bar{p}, \mu^{*}(\bar{p})\right)>0$. We have shown that his implies $\sigma_{h}^{*}(\bar{p})=0$. Now if $\sigma_{l}^{*}(\bar{p})>0$ then, by $(3)$, it follows $\mu^{*}(\bar{p})=0$. But then $q_{l}+\mu^{*}(\bar{p}) \Delta q-\bar{p}-$ $p_{c}=q_{l}-\bar{p}-p_{c}<q_{l}-\bar{p}$ so that $\left(\bar{p}, \mu^{*}(\bar{p})\right) \notin S\left(s_{c h}\right)$, which contradicts $\sigma^{*}\left(s_{c h} \mid \bar{p}, \mu^{*}(\bar{p})\right)>0$.

In order to show that $\tilde{p}<c_{h}$ implies $\Pi_{c}\left(p_{c}\right)=0$ suppose, on the contrary that, $\Pi_{c}\left(p_{c}\right)>0$. This requires that there exists some $\bar{p}$ such that $\sigma^{*}\left(s_{c h} \mid \bar{p}, \mu^{*}(\bar{p})\right)>0$ and $\sigma_{i}^{*}(\bar{p})>0$ for some $i \in\{l, h\}$. First note that $\sigma^{*}\left(s_{c h} \mid \bar{p}, \mu^{*}(\bar{p})\right)>0$ implies $\bar{p} \leq \tilde{p}$. Now suppose $\sigma_{h}^{*}(\bar{p})>0$ then $\Pi_{h}\left(\bar{p}, \mu^{*}(\bar{p}) \mid \sigma^{*}\right)=$ $\left(\sigma^{*}\left(s_{c h} \mid \bar{p}, \mu^{*}(\bar{p})\right)+\sigma^{*}\left(s_{n b} \mid \bar{p}, \mu^{*}(\bar{p})\right)\right) \bar{p}-c_{h}<0$ so that the high quality seller would make a loss and, thus, violates (2). Therefore, we have $\sigma_{h}^{*}(\bar{p})=0$. Now if $\sigma_{l}^{*}(\bar{p})>0$ then (3) implies $\mu^{*}(\bar{p})=0$ so that $\sigma^{*}\left(s_{c h} \mid \bar{p}, \mu^{*}(\bar{p})\right)=0$, which contradicts $\Pi_{c}\left(p_{c}\right)>0$.

Q.E.D.

Proof of Proposition 2: In order to express the dependence of $\tilde{\mu}$ and $\tilde{p}$ on $p_{c}$ explicitly, we write $\tilde{\mu}\left(p_{c}\right)$ and $\tilde{p}\left(p_{c}\right)$, respectively. We maximize expression (4) with respect to $p_{c}$ over the relevant domain

$$
P=\left\{p_{c} \mid p_{c} \leq \Delta q / 4 \wedge \tilde{\mu}\left(p_{c}\right) \geq \lambda \wedge \tilde{p}\left(p_{c}\right) \geq c_{h}\right\} .
$$

First, we show that (4) is increasing in $p_{c}$. Define

$$
\alpha\left(p_{c}\right) \equiv \frac{\lambda\left(\tilde{p}\left(p_{c}\right)-q_{l}\right)}{\tilde{\mu}\left(p_{c}\right) \tilde{p}\left(p_{c}\right)}
$$


so that $\Pi_{c}\left(p_{c}\right)=\alpha\left(p_{c}\right)\left(p_{c}-c_{c}\right)$. We have

$$
\alpha^{\prime}\left(p_{c}\right)=\frac{4 \lambda \Delta q^{2}}{\sqrt{\Delta q\left(\Delta q-4 p_{c}\right)}\left(q_{h}+q_{l}+\sqrt{\Delta q\left(\Delta q-4 p_{c}\right)}\right)^{2}}>0
$$

so that $\alpha\left(p_{c}\right)$ is increasing in $p_{c}$ and, hence, $\Pi_{c}\left(p_{c}\right)$ is increasing in $p_{c}$ and maximized for $\max P$.

We distinguish two cases. First, for $\lambda \leq 1 / 2$, it follows $\tilde{\mu}\left(p_{c}\right) \geq 1 / 2 \geq \lambda$. Therefore,

$$
P=\left\{p_{c} \mid p_{c} \leq \Delta q / 4 \wedge \tilde{p}\left(p_{c}\right) \geq c_{h}\right\} .
$$

Hence, $\max P$ is either $p_{c}=\Delta q / 4$ or such that $\tilde{p}\left(p_{c}\right)=c_{h}$. Because $\tilde{p}(\Delta q / 4)=$ $\left(q_{h}+q_{l}\right) / 2$, it follows that for $\lambda \leq 1 / 2$ and $c_{h} \leq\left(q_{h}+q_{l}\right) / 2$, the maximum obtains for $p_{c}=\Delta q / 4$ with

$$
\Pi_{c}^{b}=\frac{\lambda \Delta q}{2\left(q_{h}+q_{l}\right)}\left(\Delta q-4 c_{c}\right)
$$

For $\lambda \leq 1 / 2$ and $c_{h}>\left(q_{h}+q_{l}\right) / 2$ the maximum obtains for $p_{c}$ such that $\tilde{p}\left(p_{c}\right)=c_{h}$, which yields $p_{c}=\left(q_{h}-c_{h}\right)\left(c_{h}-q_{l}\right) / \Delta q$ with

$$
\Pi_{c}^{b}=\frac{\lambda\left[\left(q_{h}-c_{h}\right)\left(c_{h}-q_{l}\right)-\Delta q c_{c}\right]}{c_{h}}
$$

Second, for $\lambda>1 / 2$ we have

$$
\tilde{\mu}\left(p_{c}\right) \geq \lambda \Leftrightarrow p_{c} \leq \lambda(1-\lambda) \Delta q
$$

Since $\lambda(1-\lambda) \leq 1 / 4$ the requirement $p_{c}<\lambda(1-\lambda) \Delta q$ automatically implies $p_{c} \leq \Delta q / 4$. Hence for $\lambda>1 / 2$ we have

$$
P=\left\{p_{c} \mid p_{c} \leq \lambda(1-\lambda) \Delta q \wedge \tilde{p}\left(p_{c}\right) \geq c_{h}\right\}
$$

Because, $\tilde{p}(\lambda(1-\lambda) \Delta q)=\lambda q_{h}+(1-\lambda) q_{l}$, which by assumption is smaller than $c_{h}$, we have $\max P=\left(q_{h}-c_{h}\right)\left(c_{h}-q_{l}\right) / \Delta q$. Note that $c_{h}>\lambda q_{h}+(1-\lambda) q_{l}$ and $\lambda>1 / 2$ implies that $c_{h}>\left(q_{h}+q_{l}\right) / 2$. It follows $\tilde{\mu}=\left(c_{h}-q_{l}\right) / \Delta q$ and

$$
\Pi_{c}^{b}=\frac{\lambda\left[\left(q_{h}-c_{h}\right)\left(c_{h}-q_{l}\right)-\Delta q c_{c}\right]}{c_{h}}
$$


Proof of Proposition 3 Fix some $p_{c}<q_{h}-c_{h}$. By certifying, seller $q_{h}$ guarantees himself the payoff $\Pi_{h}(c)=q_{h}-c_{h}-p_{c}>0$. Hence, in any equilibrium of the subgame $\Gamma\left(p_{c}\right)$ seller $q_{h}$ must obtain a payoff of at least $\Pi_{h}(c)>0$.

Now suppose that there exists some equilibrium in which $\sigma_{h}(c)<1$. Then, by $(5)$ there exists some price $\tilde{p}$ such that $\sigma_{h}(\tilde{p})>0$. For $\tilde{p}$ to be optimal, it is required that $\Pi_{h}\left(\tilde{p}, \mu^{*}(\tilde{p}) \mid \sigma^{*}\right)=\tilde{p} \sigma\left(s_{n b} \mid \tilde{p}, \mu^{*}(\tilde{p})\right)-c_{h} \geq \Pi_{h}(c)>0$. This implies $\Pi_{l}\left(\tilde{p}, \mu^{*}(\tilde{p}) \mid \sigma^{*}\right)=\tilde{p} \sigma\left(s_{n b} \mid \tilde{p}, \mu^{*}(\tilde{p})\right)>c_{h}$ so that the equilibrium payoff of seller $q_{l}$ is $\Pi_{l}^{*}>c_{h}>\bar{q}$. Consequently, $\sigma_{l}^{*}(p)=0$ for any $p<\bar{q}$ and therefore

$$
\sum_{p \geq \bar{q}} \sigma_{l}^{*}(p)=1
$$

But if $\sigma_{l}^{*}(p)>0$ then we must have $p \sigma\left(s_{n b} \mid p, \mu^{*}(p)\right)>c_{h}$. This requires $\sigma\left(s_{n b} \mid p, \mu^{*}(p)\right)>0$. Therefore, $s_{n b}$ must be an optimal response given price $p$ and belief $\mu^{*}(p)$. Hence, $\mu^{*}(p) \Delta q+q_{l} \geq p>c_{h}>\lambda \Delta q+q_{l}$. As a result, $\mu^{*}(p)>\lambda$ and, due to $(3)$, it holds $\sigma_{h}^{*}(p)>\sigma_{l}^{*}(p)$ for any $\sigma_{l}^{*}(p)>0$. Together with (10) we arrive at the contradiction

$$
\sum_{p \geq \bar{q}} \sigma_{h}^{*}(p)>\sum_{p \geq \bar{q}} \sigma_{l}^{*}(p)=1
$$

It is straightforward to verify that for $p_{c} \leq q_{h}-c_{h}$, the strategies $\sigma_{h}(c)=1$, $\sigma_{l}\left(q_{l}\right)=1, \sigma^{*}\left(s_{n n} \mid p, \mu\right)=1$ whenever $\mu \Delta q+q_{l} \geq p$ and zero otherwise together with $\mu^{*}(p)=q_{l}$ constitute an equilibrium that sustains the equilibrium outcome.

For $p_{c}>q_{h}-c_{h}$, certification would yield seller $q_{h}$ a negative payoff: $\Pi_{h}(c)=q_{h}-c_{h}-p_{c}<0$. Certification would yield seller $q_{l}$ a payoff $\Pi_{l}(c)=$ $q_{l}-p_{c}<q_{l}$, whereas seller $q_{l}$ could guarantee himself the payoff $q_{l}$ by not certifying.

Q.E.D.

Proof of Proposition 4: First, suppose there exists an equilibrium in which the payoff of the certifier, $\Pi_{c}^{*}$, is strictly smaller than $\lambda\left(q_{h}-c_{h}-c_{c}\right)$. That is, $\delta=\lambda\left(q_{h}-c_{h}-c_{c}\right)-\Pi_{c}^{*}>0$. Now note that the price $p_{c}=$ $q_{h}-c_{h}-\delta / 2<q_{h}-c_{h}$ yields the certifier a payoff $\lambda\left(q_{h}-c_{h}+\delta / 2\right)>\Pi_{c}^{*}$, because Proposition 3 shows that its subgame $\Gamma\left(p_{c}\right)$ has the unique outcome that seller $q_{h}$ always certifies and seller $q_{l}$ does not. Second, note that the 
certifier cannot obtain a profit that exceeds $\lambda\left(q_{h}-c_{h}-c_{c}\right)$, because it would require that the price of certification exceeds $q_{h}-c_{h}$ or that the low quality seller certifies with a strictly positive probability. Hence, in any equilibrium the certifier obtains the payoff $\lambda\left(q_{h}-c_{h}-c_{c}\right)$. According to Proposition 3 the certifier may become this payoff only for $p_{c}=q_{h}-c_{h}$ with $\sigma_{h}(c)=1$. Q.E.D.

Proof of Proposition 5: For $\lambda \leq 1 / 2$ and $c_{h} \leq\left(q_{h}+q_{l}\right) / 2$ we have $\Pi_{c}^{s}=\lambda\left(q_{h}-c_{h}-c_{c}\right) \geq \lambda\left(q_{h}-c_{h}-c_{c}\right) \frac{q_{h}-q_{l}}{q_{h}+q_{l}} \geq \lambda\left(q_{h}-\left(q_{h}+q_{l}\right) / 2-c_{c}\right) \frac{q_{h}-q_{l}}{q_{h}+q_{l}}=$ $\lambda\left(q_{h}-q_{l}-2 c_{c}\right) \frac{q_{h}-q_{l}}{2\left(q_{h}+q_{l}\right)} \geq \lambda\left(q_{h}-q_{l}-4 c_{c}\right) \frac{q_{h}-q_{l}}{2\left(q_{h}+q_{l}\right)}=\Pi_{s}^{b}$, where the second inequality uses $c_{h} \leq\left(q_{h}+q_{l}\right) / 2$.

For $\lambda>1 / 2$ or $c_{h}>\left(q_{h}+q_{l}\right) / 2$ it follows that $\Pi_{c}^{b}=\frac{\lambda\left[\left(q_{h}-c_{h}\right)\left(c_{h}-q_{l}\right)-\Delta q c_{c}\right]}{c_{h}}<$ $\frac{\lambda\left[\left(q_{h}-c_{h}\right)\left(c_{h}-q_{l}\right)-\left(c_{h}-q_{l}\right) c_{c}\right]}{c_{h}}=\lambda\left(q_{h}-c_{h}-c_{c}\right) \frac{c_{h}-q_{l}}{c_{h}} \leq \lambda\left(q_{h}-c_{h}-c_{c}\right)=\Pi_{b}^{s}$, where the first inequality uses $q_{h}>c_{h}$.

Q.E.D.

Proof of Lemma 5: For $\lambda>1 / 2$ or $c_{h}>\left(q_{h}+q_{l}\right) / 2$, it follows

$$
x_{c}^{b}=\left(\lambda+(1-\lambda) \sigma_{l}^{*}(\tilde{p})\right) \sigma\left(s_{c h} \mid \tilde{p}, \mu_{h}^{*}\right)=\lambda \frac{\Delta q}{c_{h}} \leq \lambda=x_{c}^{s},
$$

where the inequality obtains from $q_{h}-c_{h}-c_{c}>q_{l} \Rightarrow \Delta q<c_{h}+c_{c}<c_{h}$.

For $\lambda \leq 1 / 2$ and $c_{h} \leq\left(q_{h}+q_{l}\right) / 2$, it follows

$$
x_{c}^{b}=\left(\lambda+(1-\lambda) \sigma_{l}^{*}(\tilde{p})\right) \sigma\left(s_{c h} \mid \tilde{p}, \mu_{h}^{*}\right)=\lambda \frac{2 \Delta q}{q_{h}+q_{l}} .
$$

Hence, $x_{c}^{b}<x_{c}^{s}$ if and only if $2 \Delta q<q_{h}+q_{l}$. This yields the condition $q_{h}<3 q_{l}$. Q.E.D.

Proof of Proposition 6: Due to Lemma 5 we need only check for the case $\lambda \leq 1 / 2$ and $c_{h} \leq\left(q_{h}+q_{l}\right) / 2$ and $q_{h}<3 q_{l}$. According to Proposition 2 the certifier in this case makes non-negative profits exactly when $p_{c}^{b}=$ $\Delta q / 4 \geq c_{c}$. The differences in social welfare for this case is

$$
\begin{aligned}
\Delta W F & =\lambda \frac{\Delta q}{q_{h}+q_{l}} q_{l}+\lambda\left(\frac{2 \Delta q}{q_{h}+q_{l}}-1\right) c_{c} \\
& =\frac{\lambda}{q_{h}+q_{l}}\left(\Delta q q_{l}-\left(3 q_{l}-q_{h}\right) c_{c}\right) \\
& \geq \frac{\lambda}{q_{h}+q_{l}}\left(\Delta q q_{l}-\left(3 q_{l}-q_{h}\right) \Delta q / 4\right) \\
& =\lambda \Delta q / 4>0 .
\end{aligned}
$$


Q.E.D.

\section{Literature}

Akerlof, G., 1970, The Market for Lemons: Quality Uncertainty and the Market Mechanism, Quarterly Journal of Economics, 84, 488-500.

Albano, G. and A. Lizzeri, 2001, Strategic Certification and Provision of Quality, International Economic Review, 42, 267-284.

Bester, H. and K. Ritzberger (2001), Strategic Pricing, Signalling, and Costly Information Acquisition, International Journal of Industrial Organization, $19,1347-1361$

Biglaiser, G., 1993, Middlemen as Experts, RAND Journal of Economics 24, 212-223.

Bouvard, M. and R. Levy (2009), Humouring Both Parties: a Model of Two Sided Reputation, mimeo, Universite de Toulouse I

De, S. and P. Nabar (1991), Economic Implications of Imperfect Quality Certification, Economics Letters, 37, 333-337

Emons, W. (1997), Credence Goods and Fraudulent Experts, The RAND Journal of Economics, 28, 107-119

Fasten, E. and D. Hofmann (2010), Two-sided Certification: The Market for Rating Agencies, mimeo, Humboldt University of Berlin

Faulhaber, G. and D. Yao (1989), "Fly-by-Night Firms and the Market for Product Review", The Journal of Industrial Economics, 38, 65-77

Guerra, G. (2001), Certification Disclosure and Informational Efficiency: A Case for Ordered Ranking of Levels, mimeo, University of Oxford

Hvide, H.(2004), A Theory of Certification with an Application to the Market for Auditing Services, mimeo, Norvegian School of Economics, Bergen Lizzeri, A., 1999, Information Revelation and Certification Intermediaries, RAND Journal of Economics 30, 214-231.

Mueller, F., Stahl, K. and F. Wachtler (2008), Upstream Relationships in the Automotive Industry: A Contractual Perspective, mimeo, University of Mannheim

Peyrache, E. and L. Quesada (2004), Strategic Certification, mimeo, University of Wisconsin-Madison 
Strausz, R. (2005), Honest Certification and the Threat of Capture, International Journal of Industrial Organization, 23, 45-62

Strausz, R. (2010), Separating Equilibria with Imperfect Certification, mimeo Humboldt Universität Berlin.

Viscusi (1978) A Note on "Lemons" Markets with Quality Certification, Bell Journal of Economics 9, 277-279

Wolinsky (1993) Competition in a Market for Informed Experts, RAND Journal of Economics 24, 380-398 277-279 